\title{
ANNUAL WATER-RESOURCES REVIEW, WHITE SANDS MISSILE RANGE, NEW MEXICO, 1982
}

BY R. R. CRUZ

U.S. GEOLOGICAL SURVEY

OPEN-FILE REPORT 83-695

PREPARED IN COOPERATION WITH

WHITE SANDS MISSILE RANGE

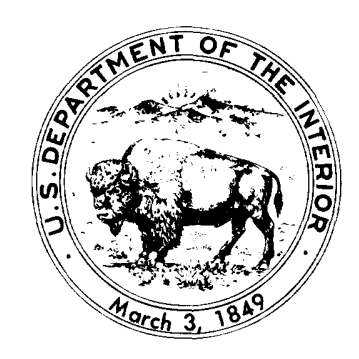

ALBUQUERQUE, NEW MEXICO

1983 


\section{UNITED STATES DEPARTMENT OF THE INTERIOR \\ WILLIAM P. CLARK, Secretary \\ GEOLOGICAL SURVEY \\ Dallas L. Peck, Di rector}

For additional information write to:

District Chief

U.S. Geologica1 Survey Water Resources Division 505 Marquette NW, Room 720

Albuquerque, New Mexico 87102
For sale by:

Open-File Services Section Branch of Distribution U.S. Geological Survey, MS 306 Box 25425, Denver Federal Center Denver, Colorado 80225 (303) 234-5888 


\section{CONTENTS}

Abstract -

Page

Introduction

We11-numbering system - 2

Data-collection program 3

Ground-water pumpage - 3

Water-level measurements in supply wells 8

Water-leve1 measurements in test we1ls, observation we11s, and boreholes - 8

Chemical quality 8

Miscellaneous observations

Selected references - 18

\section{ILLUSTRATIONS}

Figure 1. Location map of White Sands Missile Range and areas of hydrologic observations - 4

2. Map showing location of supply wells, test we11s, observation wells, and boreholes in the Post Headquarters and adjacent areas

3. Diagram showing location of we11s in Mockingbird Gap and $\mathrm{NW}-30$ areas

4. Map showing location of supply wells, Stallion Range Center- 6

5. Graphs showing monthly and yearly pumpage in the Post Headquarters well field, 1968-82, and water-level fluctuations in Main Gate well

6-8. Graphs showing water levels and specific-conductance values for period of record available in supply wells:

6. $10 \mathrm{~A}, 11,13$, and 15 9

7. 16,17 , and 18

8. $19,20,21$, and 22

9. Graph showing water-level fluctuations in test wells $\mathrm{T}-7, \mathrm{~T}-8, \mathrm{~T}-10$, and $\mathrm{T}-11$ for $1973-82$

10. Map showing specific-conductance values and $\mathrm{pH}$ values of water from selected wells, Post Headquarters and Range areas, 1982 


\section{ILLUSTRATIONS}

Figure 11. Map showing location of wells and springs sampled in

Page northern White Sands Missile Range

12. Map showing location of vertical electrical soundings

in Ash Canyon area -

13. Map showing location of vertical electrical soundings

in Rhodes Canyon area

\section{TABLES}

Table 1. Test wells drilled by military in Post Headquarters area, White Sands Missile Range, 1982

2. Depth to water in supply wells, Post Headquarters and Range areas, 1982

3. Depth to water in test and observation wells, Post Headquarters and Range areas, 1982

4. Depth to water in boreholes, Post Headquarters and adjacent areas, 1982

5. Specific-conductance values of water samples collected from supp1y we11s, test we11s, and observation we1ls, 1982

6. Major chemical-constituent analyses of water from selected wells, White Sands Missile Range --

7. Chemical analyses of water from selected wells and springs, northern White Sands Missile Range --_---_----

\section{CONVERSION FACTORS}

In this report, values for measurements are given in inch-pound units only. The following table contains factors for converting to International System (SI) units.

Multiply inch-pound units

foot

mile

gallon

acre-foot
By

0.3048

1.609

0.003785

1,233
To obtain SI units

meter

kilometer

cubic meter

cubic meter 


\title{
ANNUAL WATER-RESOURCES REVIEW, WHITE SANDS MISSILE RANGE, NEW MEXICO, 1982
}

BY R. R. CRUZ

\begin{abstract}
Ground-water data were collected in 1982 at White Sands Missile Range in south-central New Mexico. Depth-to-water measurements in the Post Headquarters supply wells continued to show seasonal declines. Test wells east of the Headquarters well field continued to show long-term declines as wel1 as seasonal fluctuations. The total amount of water pumped from White Sands Missile Range supply wells was 66,226,600 gallons more in 1982 than in 1981. There was a greater difference in specific-conductance between winter and summer water samples from the Post Headquarters supply wells in 1982 than there has been in the past.
\end{abstract}

\section{INTRODUCTION}

This report presents water-resources information that was collected at White Sands Missile Range during 1982 by personnel of the U.S. Geological Survey. Ground-water pumpage, water-level measurements, and chemical-quality data summarized in this report were obtained as a result of the continuing water-resources hydrologic-data-collection program sponsored by the Facilities Engineering Directorate, White Sands Missile Range.

The 1968 report and subsequent annual reports are open-file reports and are available for inspection at the District Office of the U.S. Geological Survey, Water Resources Division, in Albuquerque, New Mexico. 
We11-numbering System

Wells are located according to the system of common subdivision of sectionized land used throughout the State by the U.S. Geological Survey. The number of each well consists of four segments separated by periods and locates the well's position to the nearest 10-acre tract of land. The segments denote, respectively, the township south of the New Mexico base line, the range east of the New Mexico principal meridian, the section, and the particular 10-acre tract within the section.

The fourth segment of the number consists of three digits denoting, respectively, the quarter section or approximate 160-acre tract, the quadrant (approximately 40 acres in size) of the quarter section, and the quadrant (approximately 10 acres in size) of the 40-acre tract in which the well is located. The system of numbering quarter sections and quadrants, which is done in reading order, as well as the usual numbering of sections within a township is shown below. For example, well $22 \mathrm{~S} .4 \mathrm{E} .1 .431$ is located in the NW/1

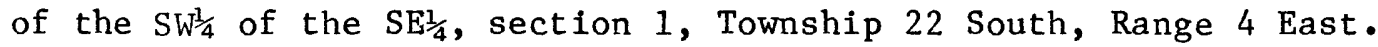

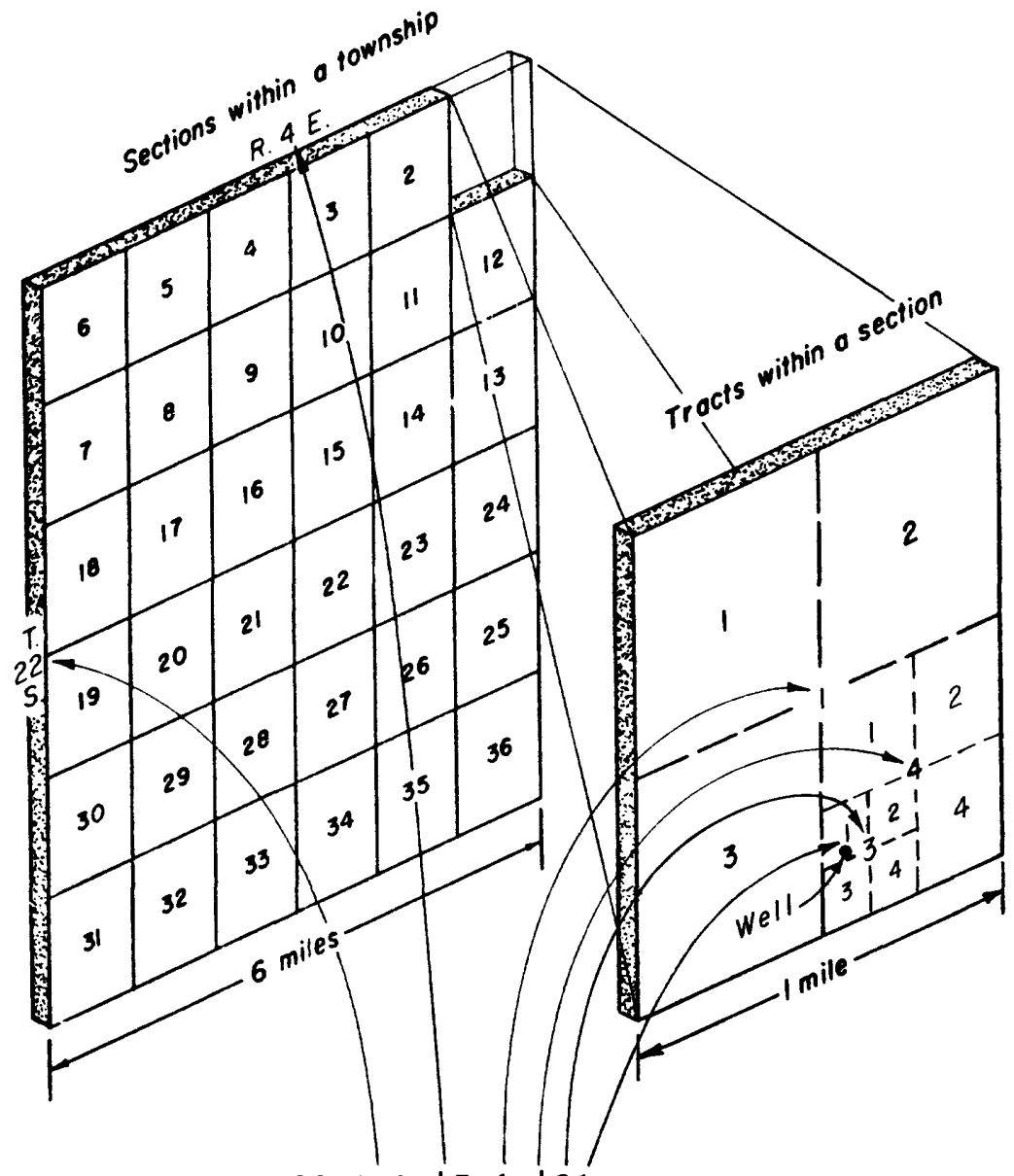

We $1122 S .4 E \cdot 1.431$ 


\section{DATA-COLLECTION PROGRAM}

The program to collect hydrologic data at White Sands Missile Range (fig. 1) has been continuous since 1953. The original program consisted of water-level observations in five test wells in the Post Headquarters area. Over the years, the program has expanded to include water-level observation points and chemical-quality sampling points in seven Range areas (Gregg, Hazardous Test, Small Missile Range, Multifunction Array Radar, NW-30 Tracking Station, Mockingbird Gap, and Stallion Range Center) and more extensive coverage in and around the Post Headquarters (figs. 2, 3, 4).

In 1982 , six test wells were drilled in the Post Headquarters area (fig. 2). Drillers' logs or lithologic logs are available for all of the test wells. Borehole-geophysical logs were run in five of the test wells drilled in 1982 (table 1). Four of the test wells will be developed and used for water-level measurements and chemical-quality sampling.

Semiannual water-level measurements were made in 17 supply wel1s, 27 test and observation wells, and 38 boreholes (tables 2, 3, and 4) in 1982. Water samples were collected for laboratory specific-conductance measurements from 30 wells in 1982 (table 5). In addition, six water samples from five wells were collected in 1982 for analysis of major chemical constituents (table 6).

\section{Ground-water Pumpage}

Total ground-water pumpage* at White Sands Missile Range in 1982, according to records provided by the Facilities Engineering Directorate, was $732,171,600$ gallons. The Post Headquarters well field produced 706,652,800 gallons; Stallion Range Center wells (SRC-1 and -2) produced 7,894,000 gallons; and Hazardous Test Area (HTA-1), Small Missile Range (SMR-1), and Multifunction Array Radar wells (MAR-1 and -2) produced 17,624,800 gallons in 1982. Total pumpage at White Sands Missile Range was 66,226,600 gallons more in 1982 than in 1981. Pumpage by month and total gallons pumped per year in the Post Headquarters well field for 1968-82 and a hydrograph for Main Gate we11 for 1968-78 are shown in figure 5. The Main Gate well is dry at a depth of 414 feet.

* The pumpage figures used in this report are to be considered as preliminary figures and may be subject to revision. 


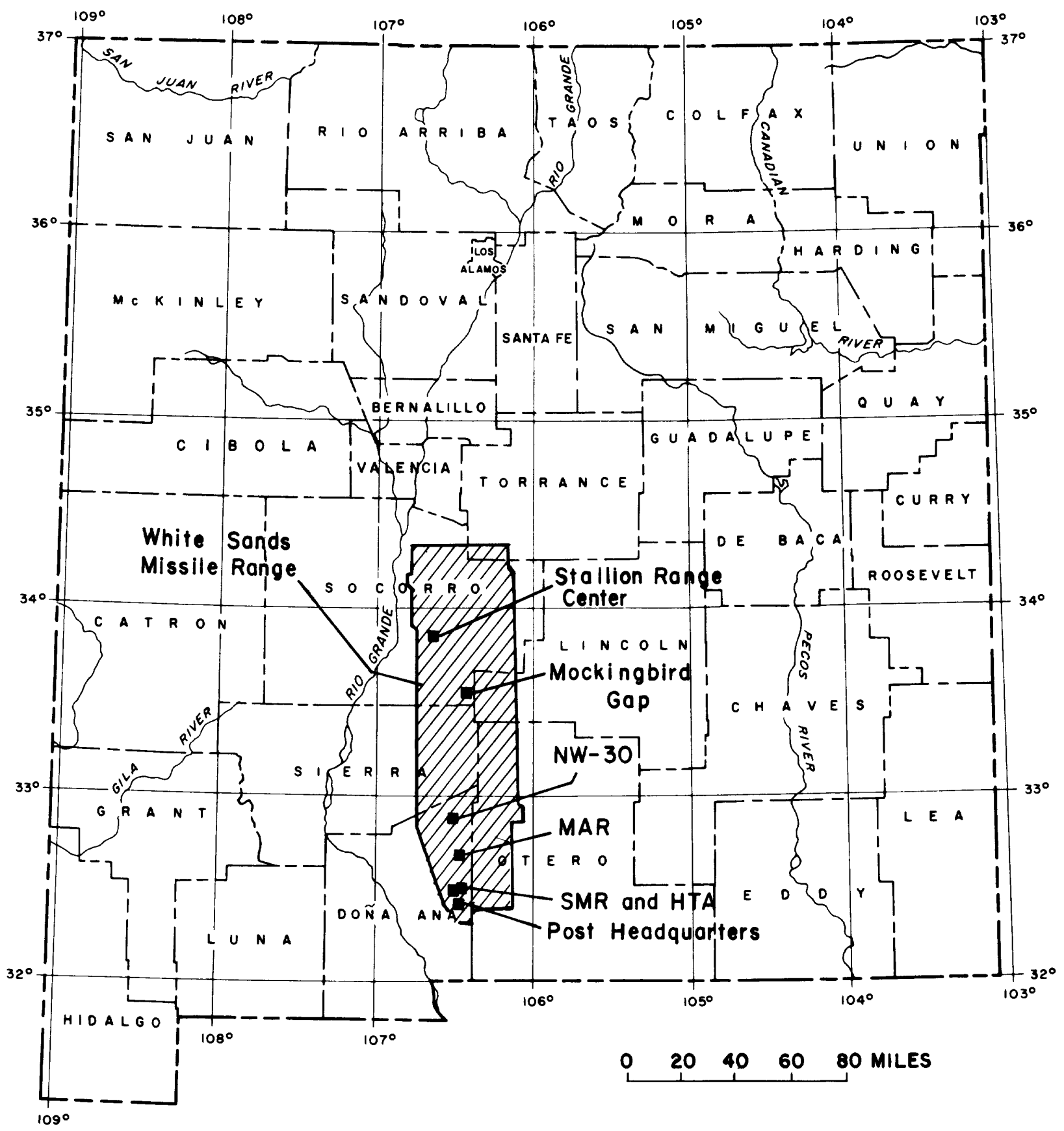

Figure 1. White Sands Missile Range and areas of hydrologic observations. 


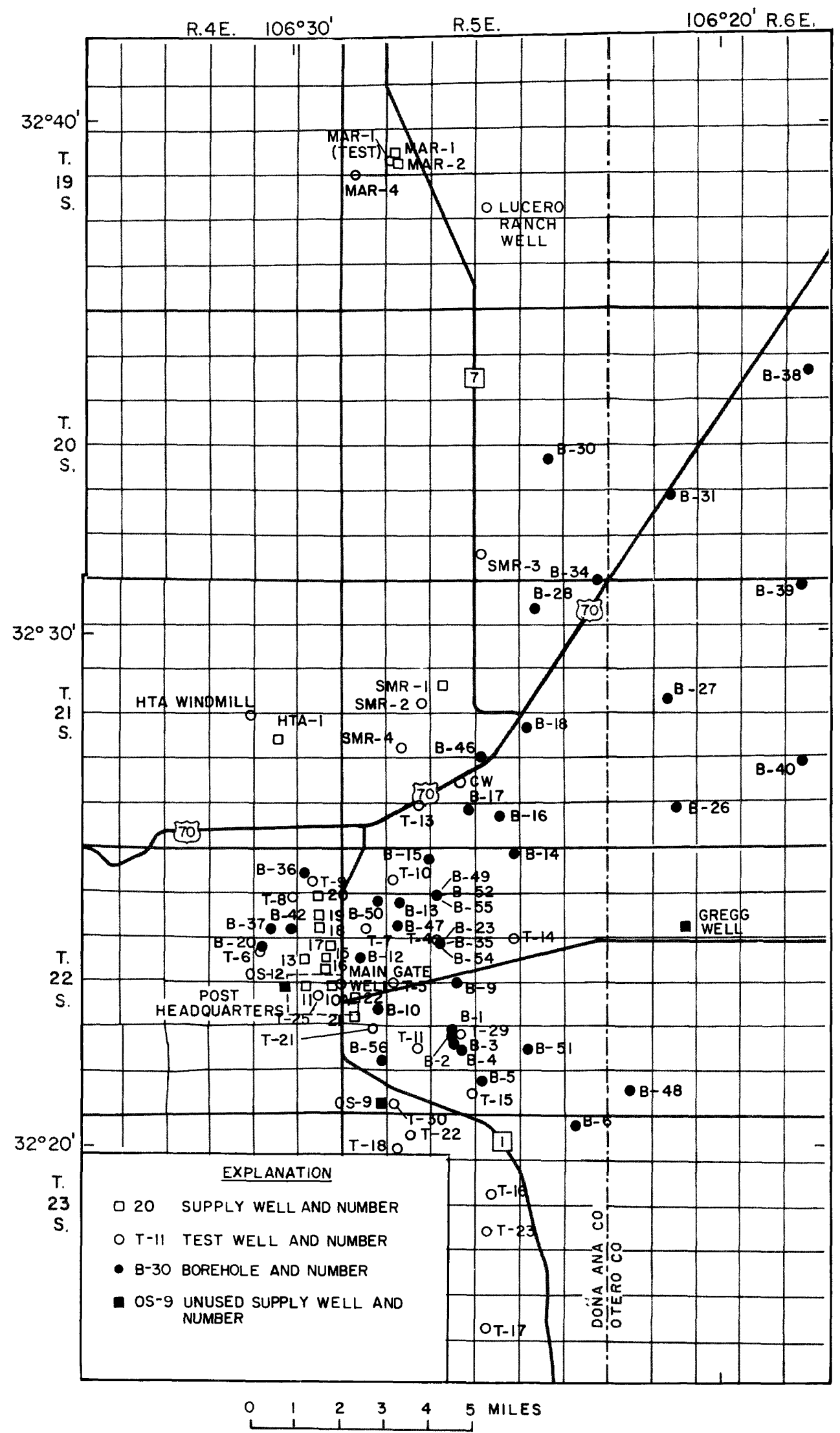

Figure 2 Location of supply wells, test wells, observation wells, and boreholes in the Post Headquarters and adjacent areas. 


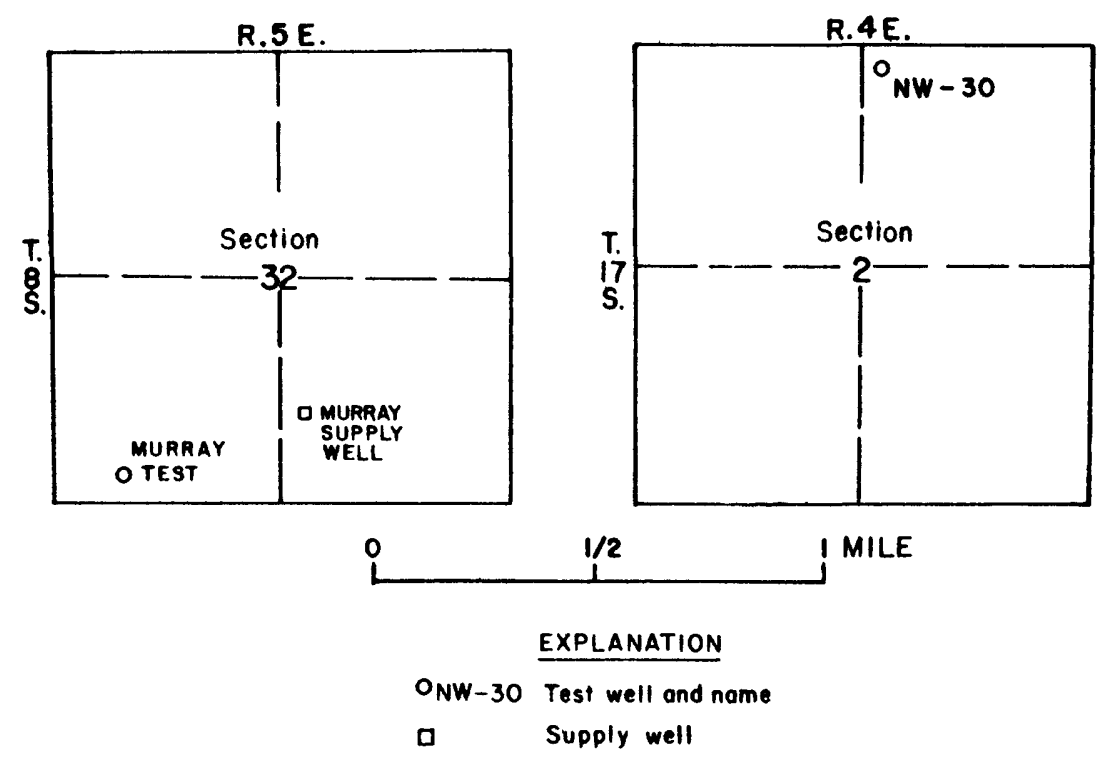

Figure 3. Location of wells in Mockingbird Gap and NW-30 areas.

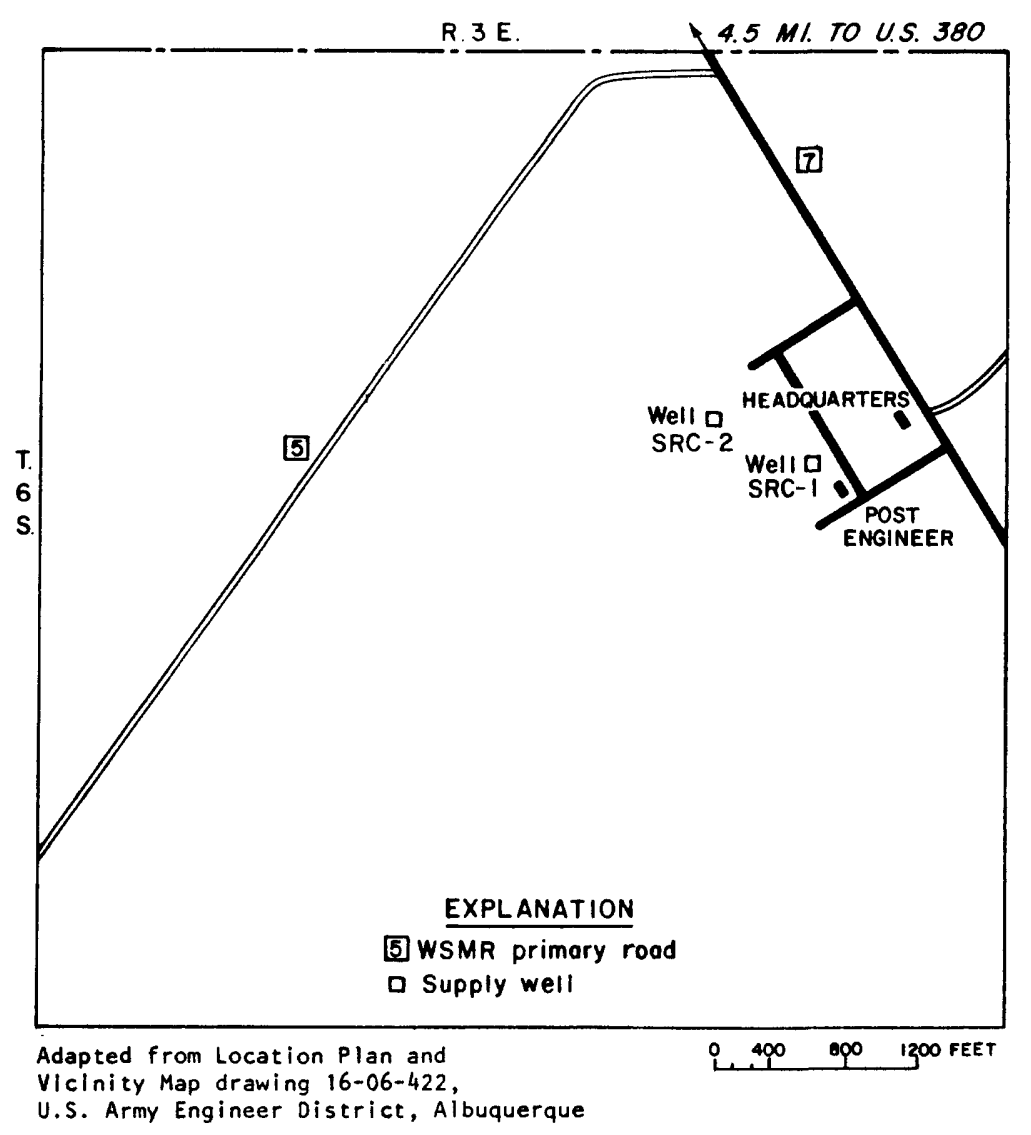

Figure 4. Location of supply wells, Stallion Range Center. 

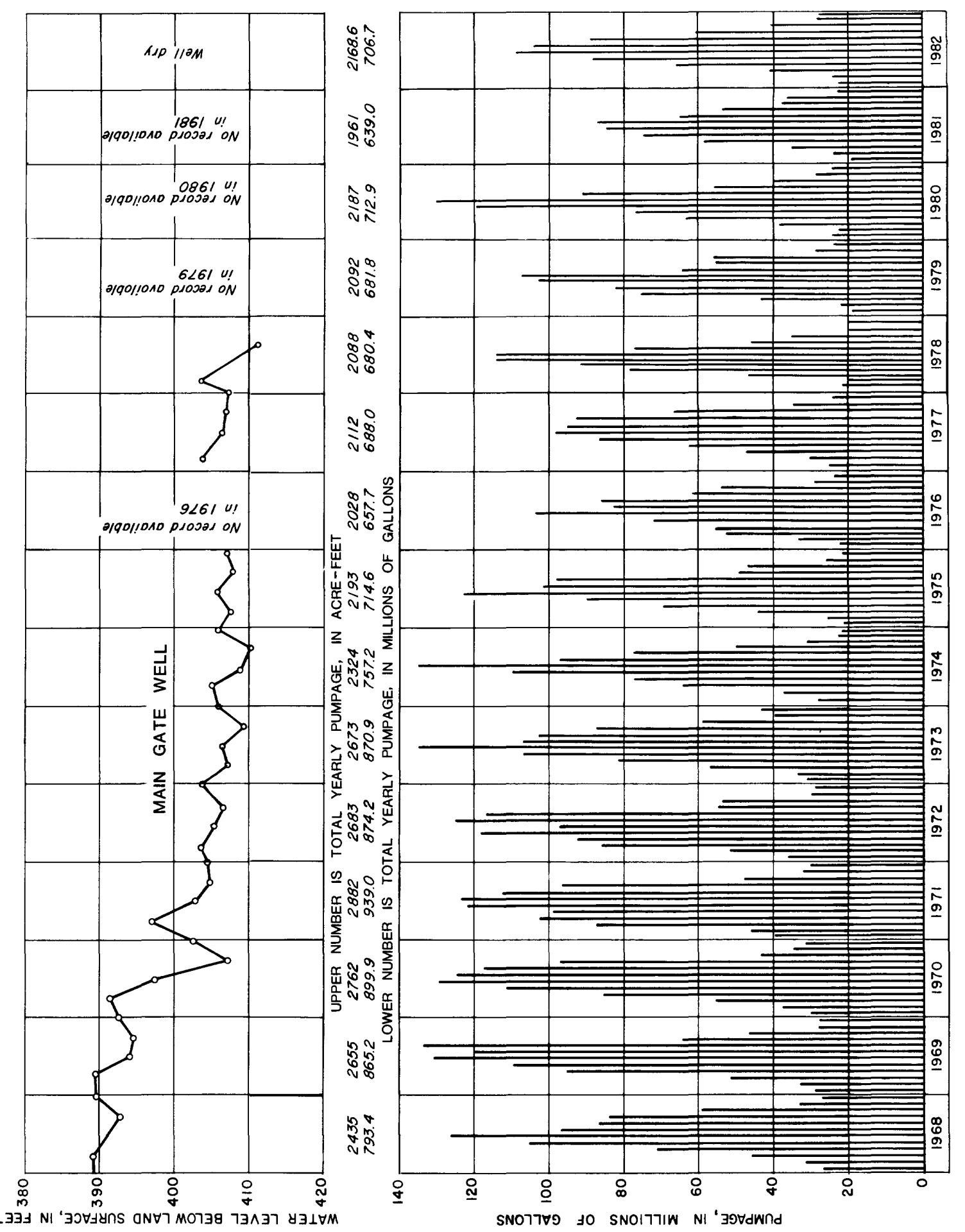

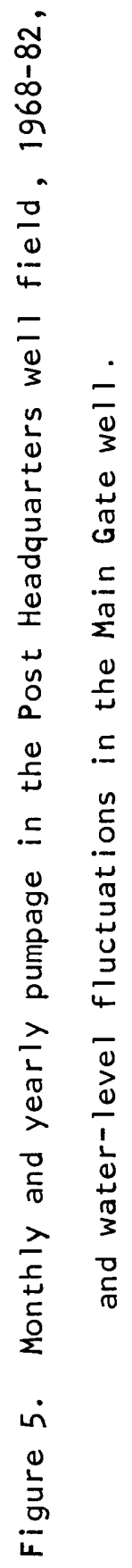


Water-level Measurements in Supply Wells

Semiannual depth-to-water measurements were made in 11 supply wells in the Post Headquarters area and in 6 supply wells in the Range areas (table 2). Seasonal fluctuations were observed in all of the supply wells in the Post Headquarters well field (figs. 6-8).

The pump was taken out of supply well 15 in April 1982; this well has not been used since June 1980. Supply well 15 was drilled to a depth of 1,010 feet and finished at 820 feet in February 1954. The water level on April 30, 1954, was 337.89 feet. A down-hole camera was lowered into supply well 15 on April 14, 1982. The water level was 430 feet below land surface and the total depth of well was 684. Water was falling through the perforation at a depth of 426 feet. A continuous water-level recorder was installed in supply well 15 in August 1982 .

Water-level Measurements in Test Wells, Observation Wells, and Boreholes

Semiannual depth-to-water measurements were made during February 1982 and August 1982 in 27 test and observations wells (table 3 ) and 38 boreholes (table 4). Four of the test wells ( $T-7, T-8, T-10$, and $T-11$ ) in the Post Headquarters area are equipped with continuous water-level recorders; hydrographs of these test wells are shown in figure 9.

\section{Chemical Quality}

Thirty-four water samples were collected from supply, test, and observation wells for laboratory analysis of specific conductance (table 5). Specific-conductance values and $\mathrm{pH}$ values for selected wells in the Post Headquarters and adjacent areas are shown in figure 10. Six water samples from five wells were collected in 1982 for analysis of major chemical constituents (table 6). 
Supply Well IOA
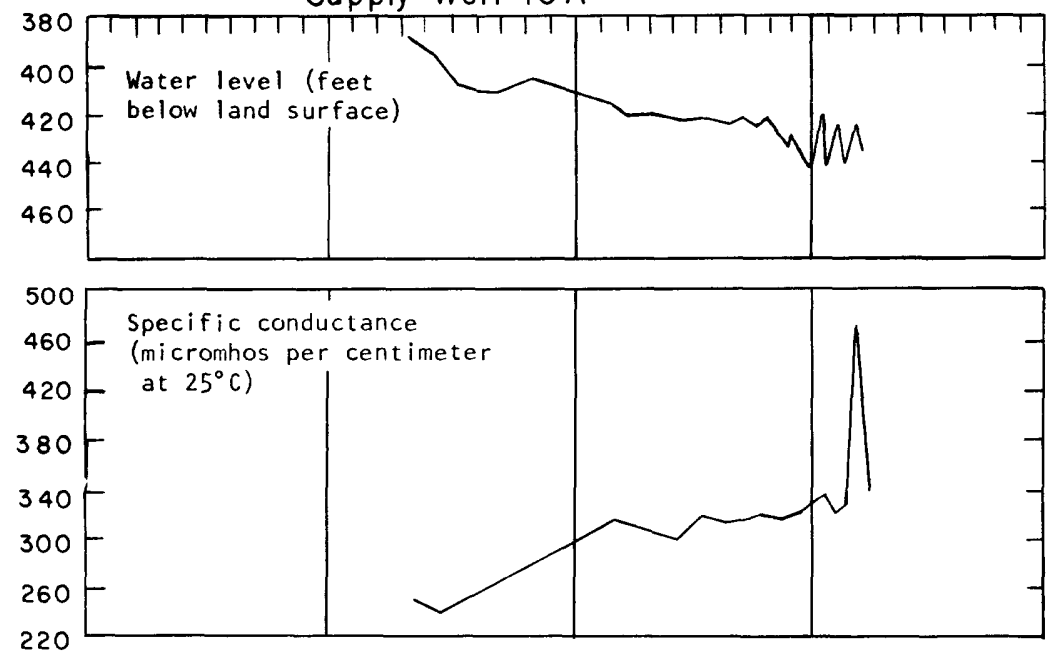

Supply Well II
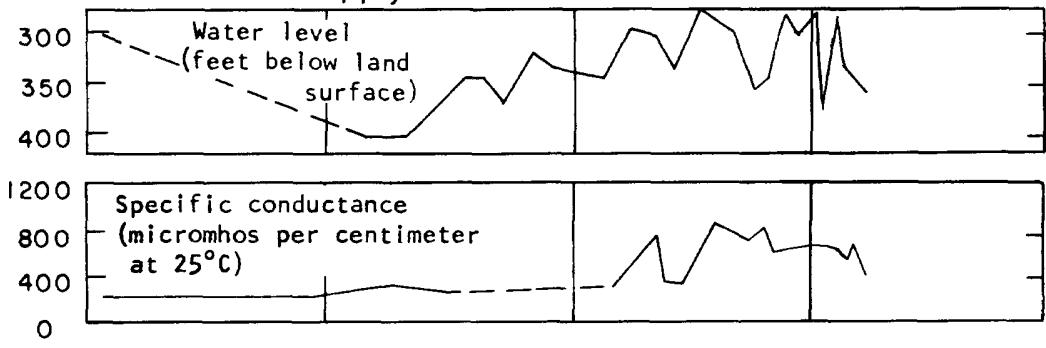

Supply Well 13
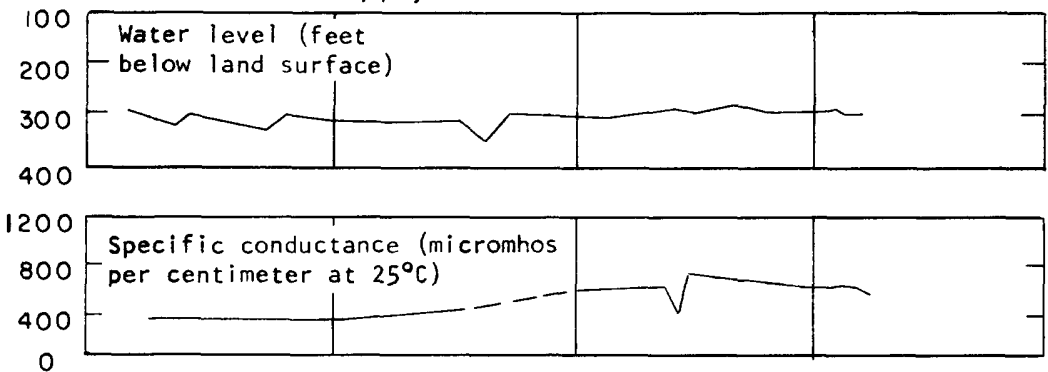

Supply Well 15

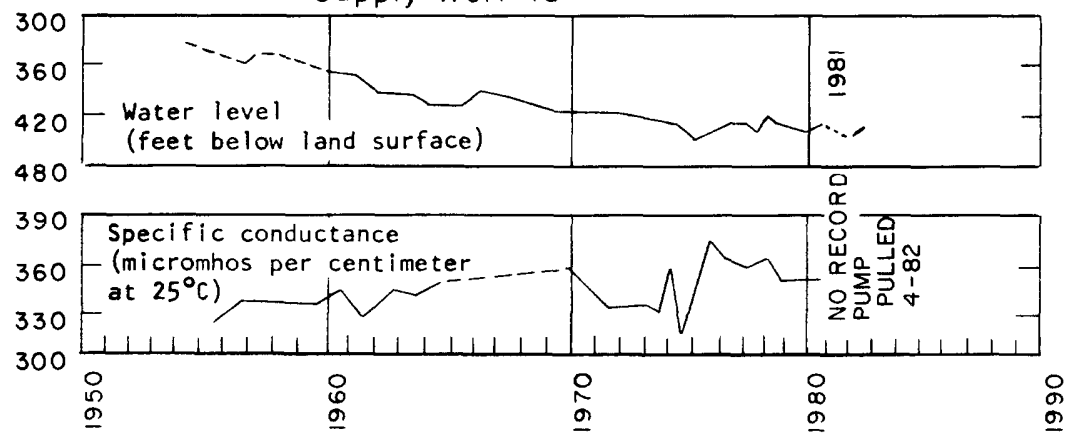

EXPLANATION

- Estimated record

Figure 6. Water levels and specific-conductance values for period of record available in supply wells $10 \mathrm{~A}, 11,13$, and 15 . 


\section{Supply Well 16}
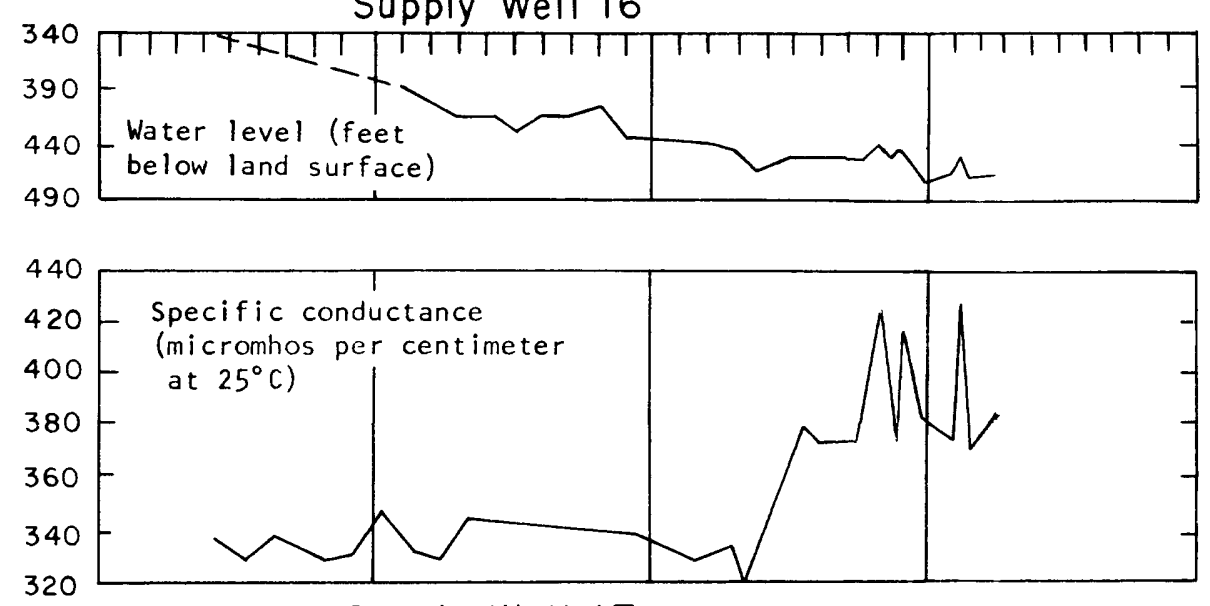

Supply Well 17
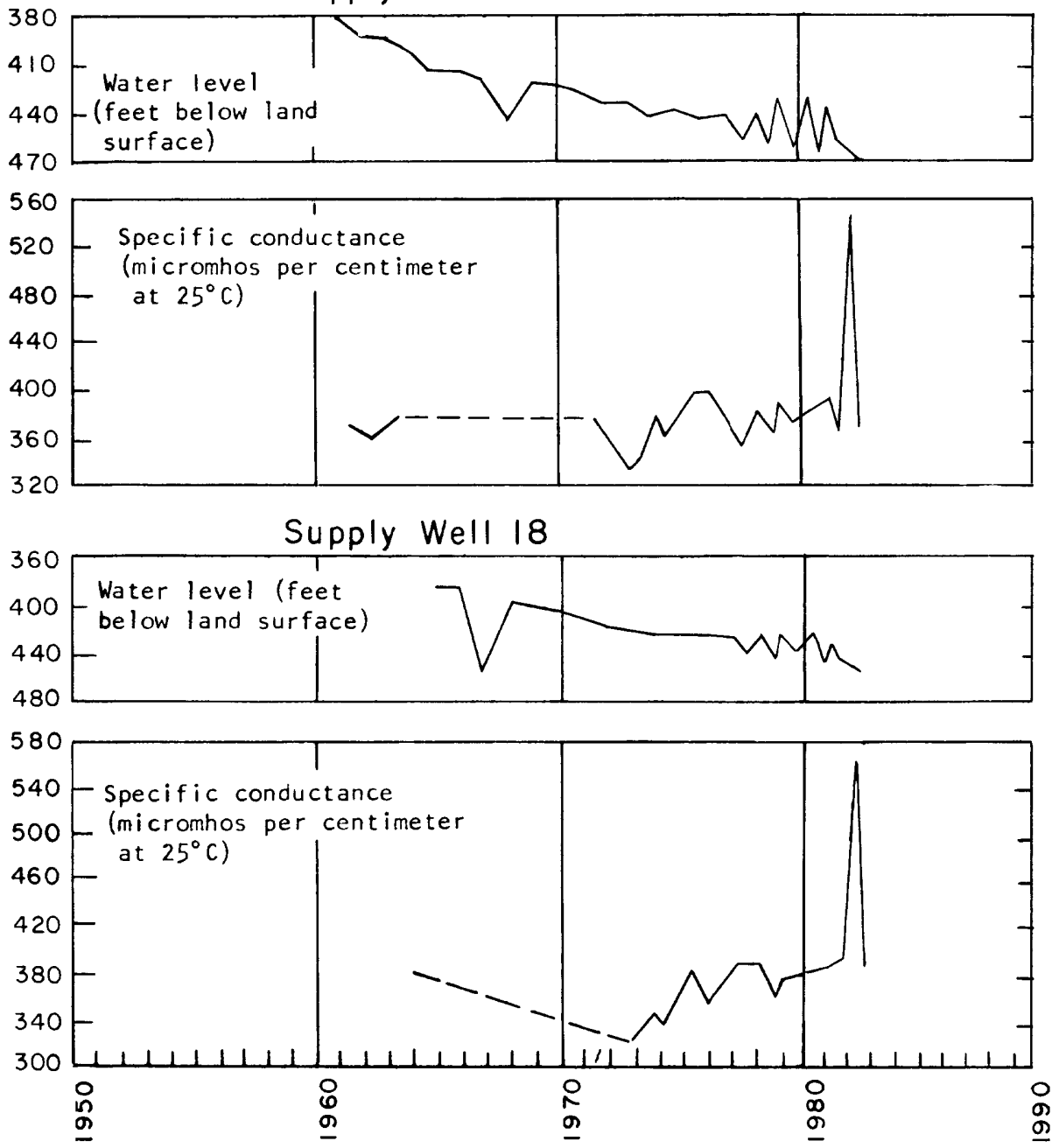

EXPLANATION

- - Estimated record

Figure 7. Water levels and specific-conductance values for period of record available in supply wells 16,17 , and 18 . 
Supply Well 19
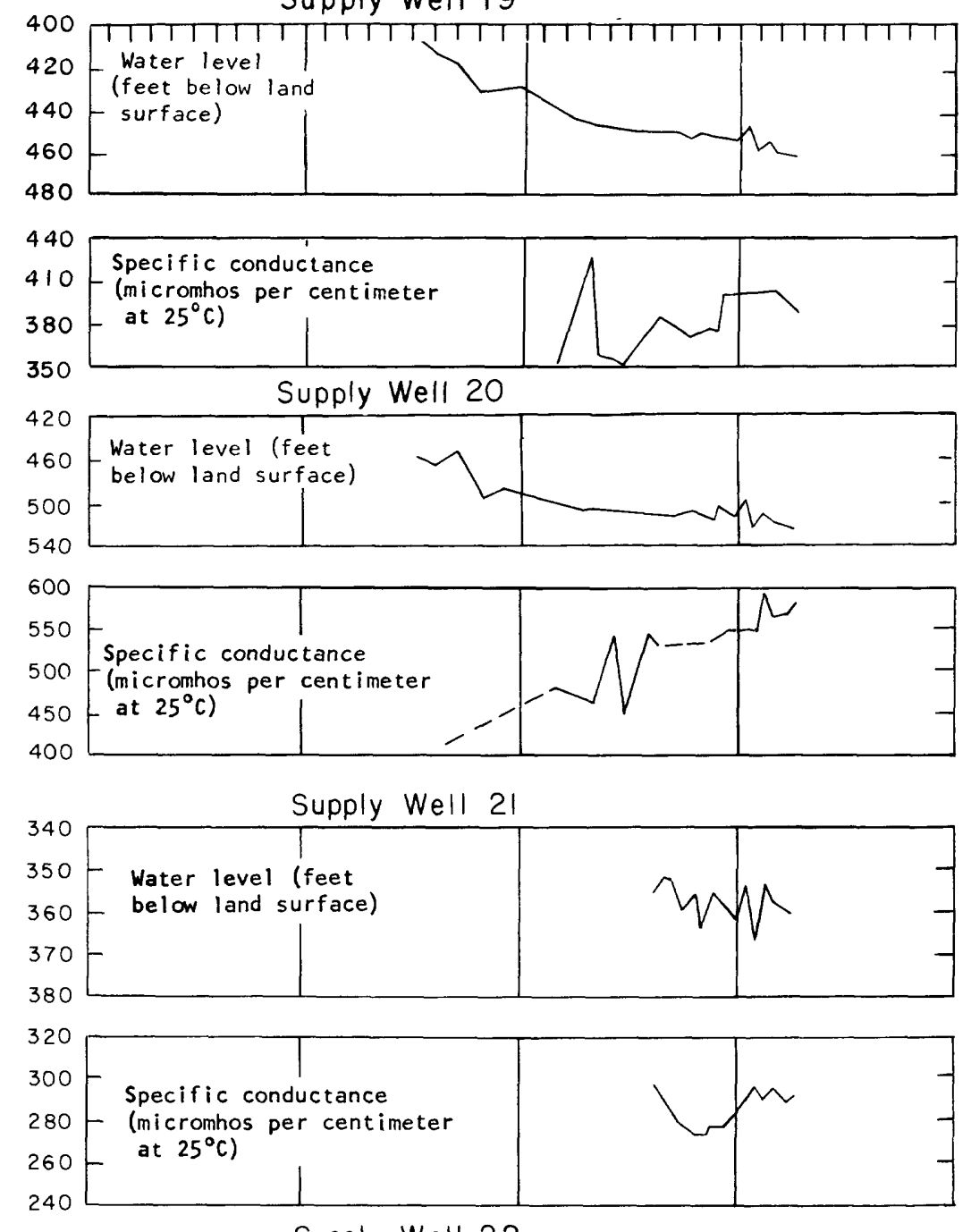

Supply Well 22
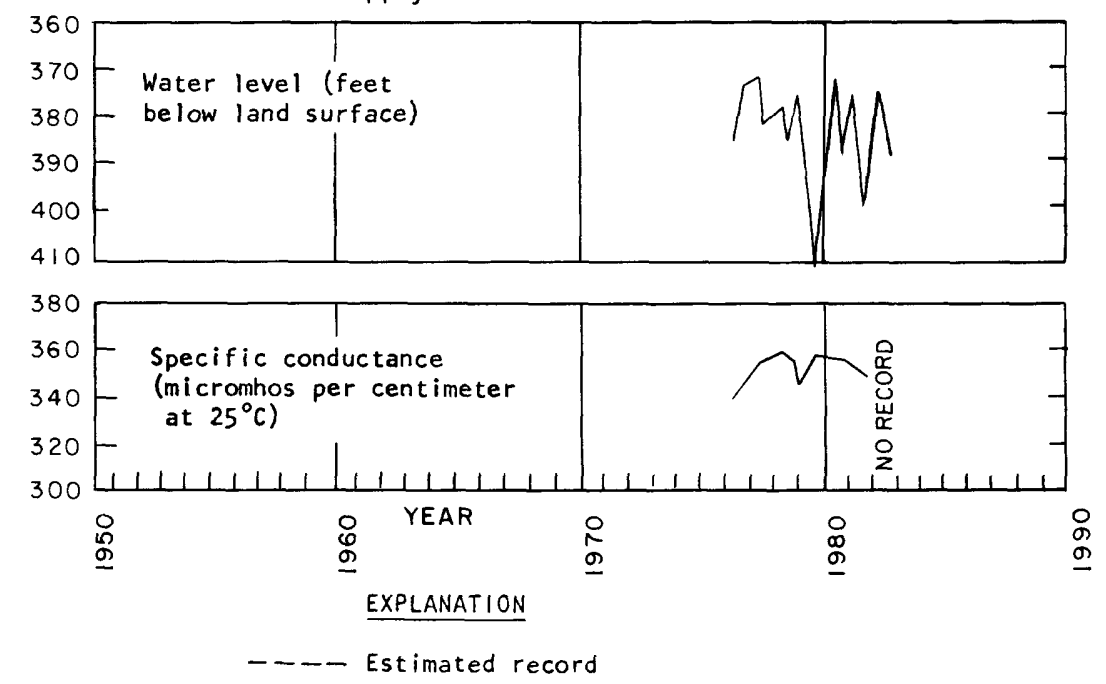

Figure 8. Water levels and specific-conductance values for period of record available in supply wells 19, 20, 21, and 22 . 


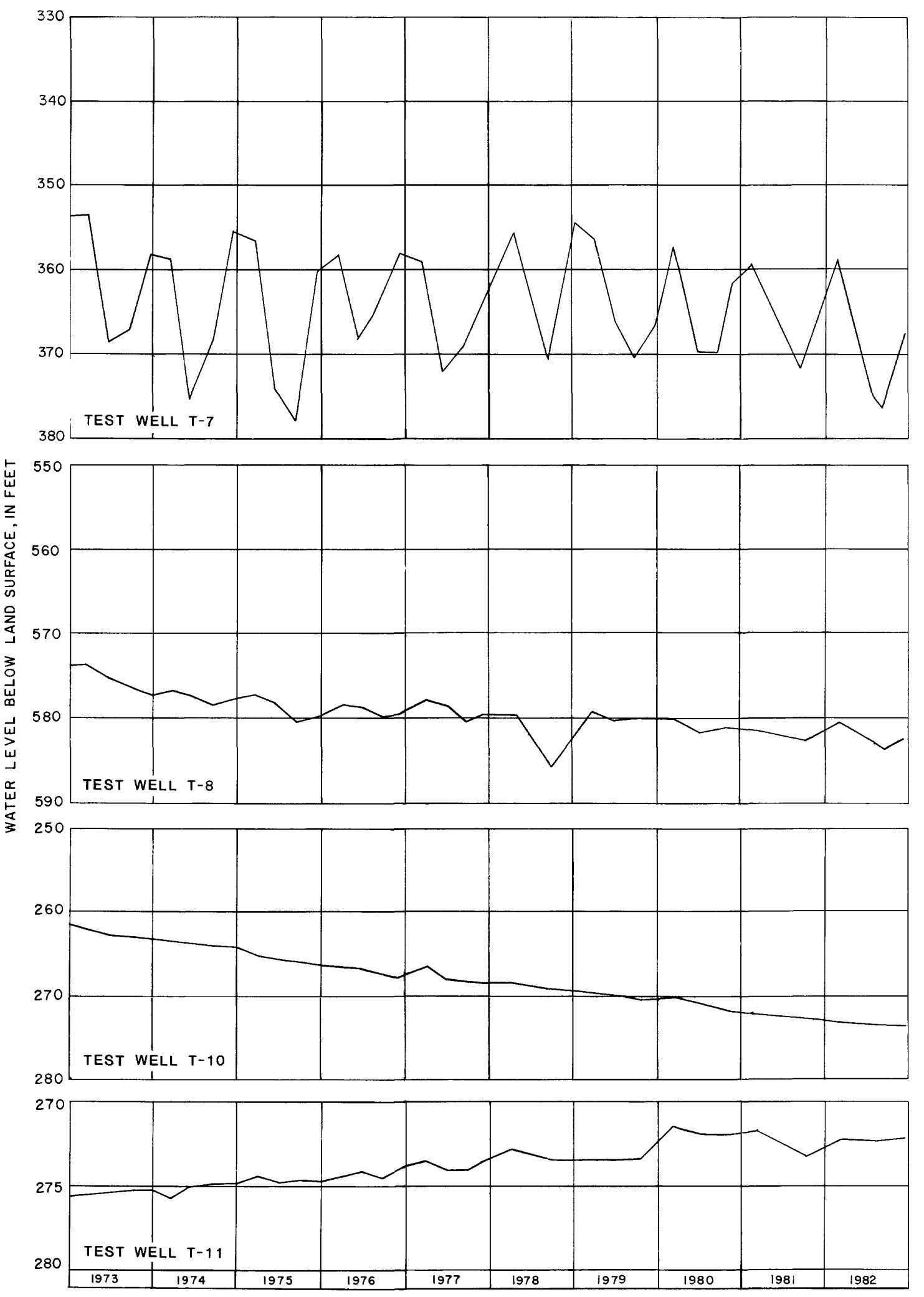

Figure 9. Water-level fluctuations in test wells $T-7, T-8, T-10$, and $T-11$ for $1973-82$. 


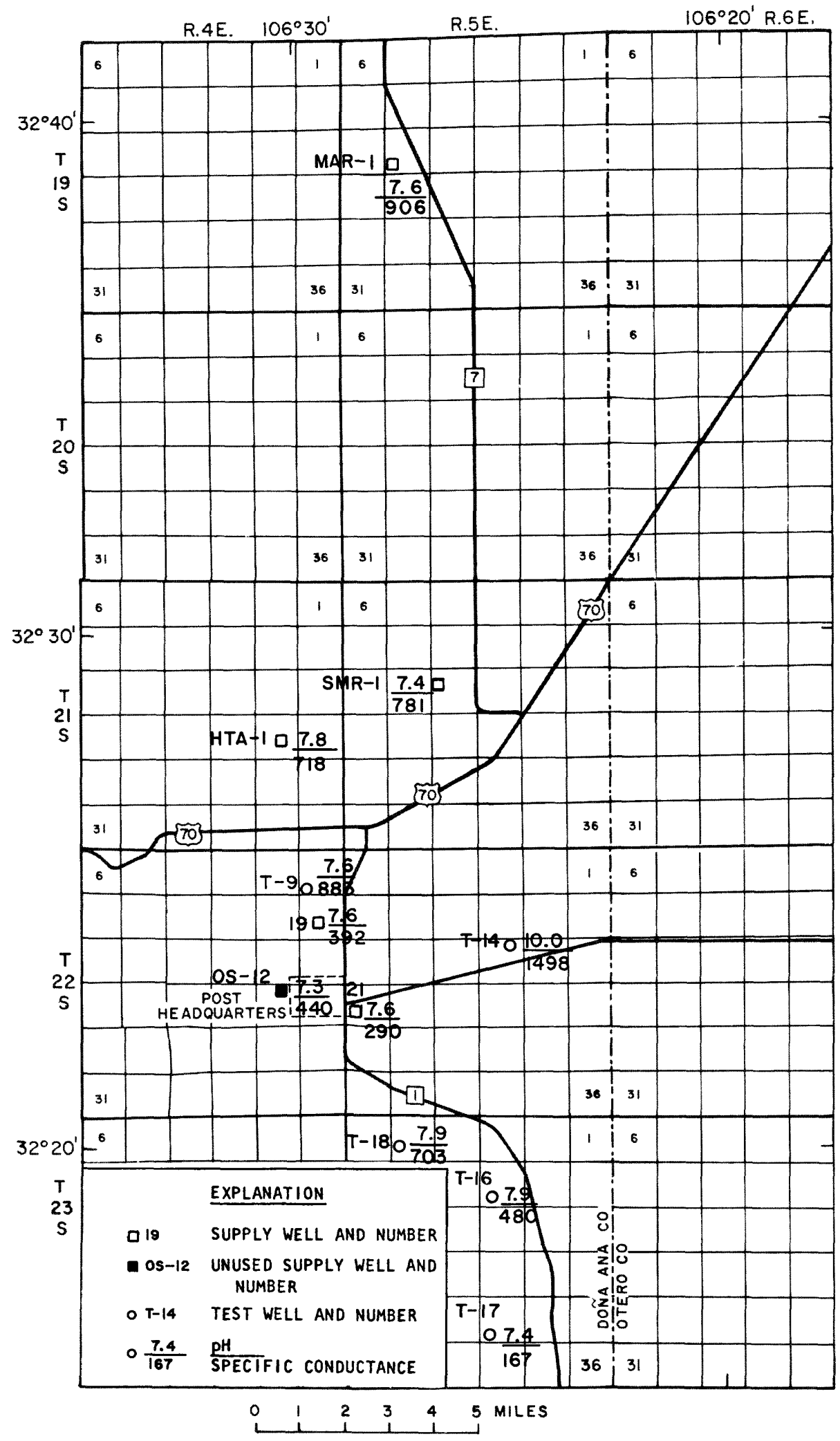

Figure 10. Specific-conductance values and $\mathrm{pH}$ values of water from selected wells, Post Headquarters and Range areas, 1982. 


\section{MISCELLANEOUS OBSERVATIONS}

Water samples were collected in the central and northern part of the Tularosa basin (fig. 11) in July and August 1982 from selected wells and springs. Five of the sampling sites were on or within 1 mile of White Sands Missile Range. Some of the sites had been sampled previously, and these analyses and the 1982 analyses are included in table 7. The 1982 analyses were rather extensive and include age dating of the water by the carbon-14 method. The older water in the area sampled comes from the deeper Permian Yeso Formation, and the younger water is from the Quaternary alluvium.

Vertical electrical soundings (VES) were made at two uprange areas in March 1982. Seven soundings were made in the vicinity of Ash Canyon on the fan slope (fig. 12). Seventeen soundings were made in the Rhodes Canyon area (fig. 13). The data from these soundings are available from the U.S. Geological Survey, Water Resources Division, Las Cruces field office on the New Mexico State University campus. 


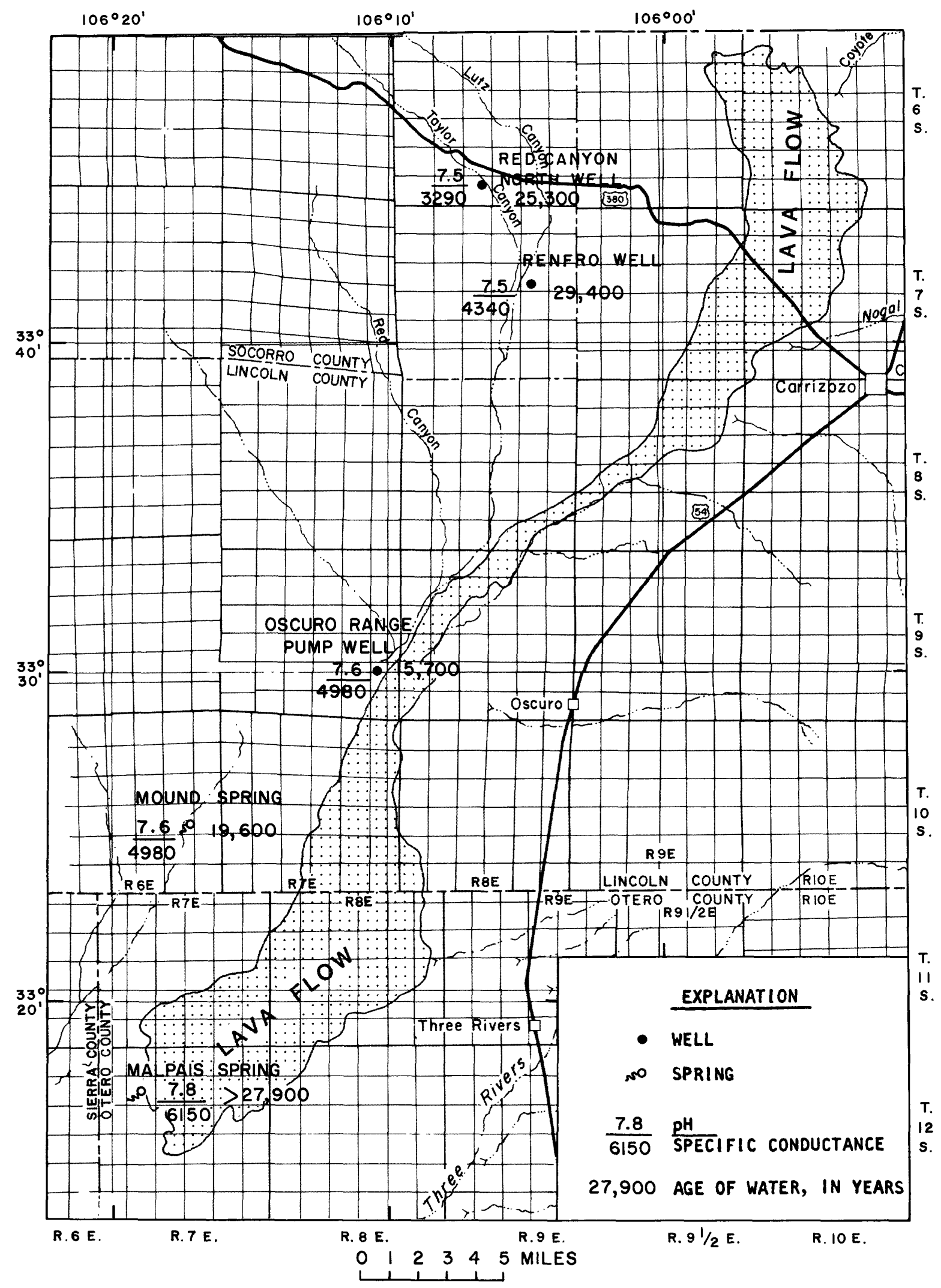

Figure 11. Location of wells and springs sampled in northern White Sands Missile Range. 


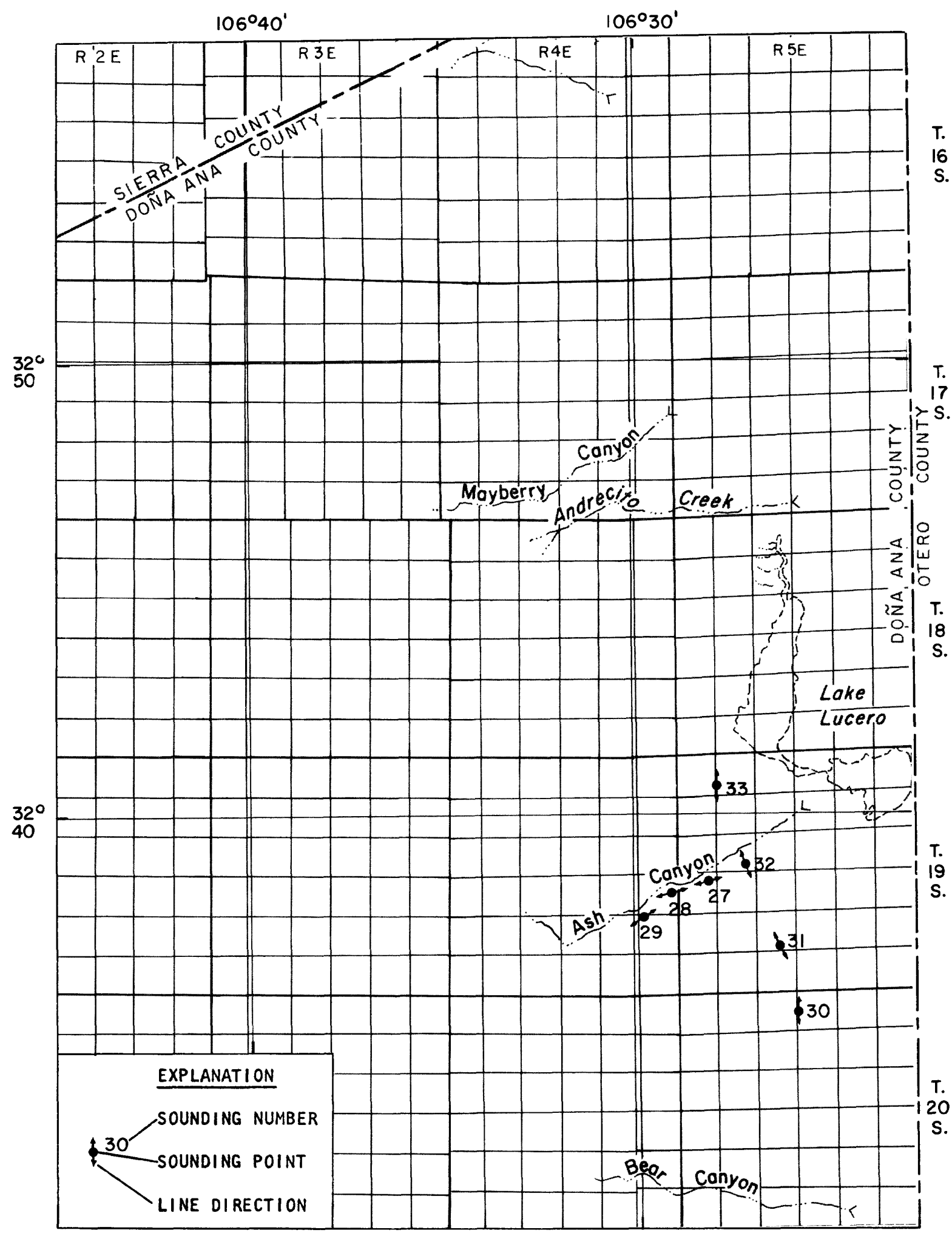
R. $2 \mathrm{E}$.
R. 3 E.
R. $4 \mathrm{E}$.
R. 5 E.

\section{\begin{tabular}{llllll}
0 & 1 & 2 & 3 & 4 & 5 \\
\hline
\end{tabular}}

Figure 12. Location of vertical electrical soundings in Ash Canyon area. 


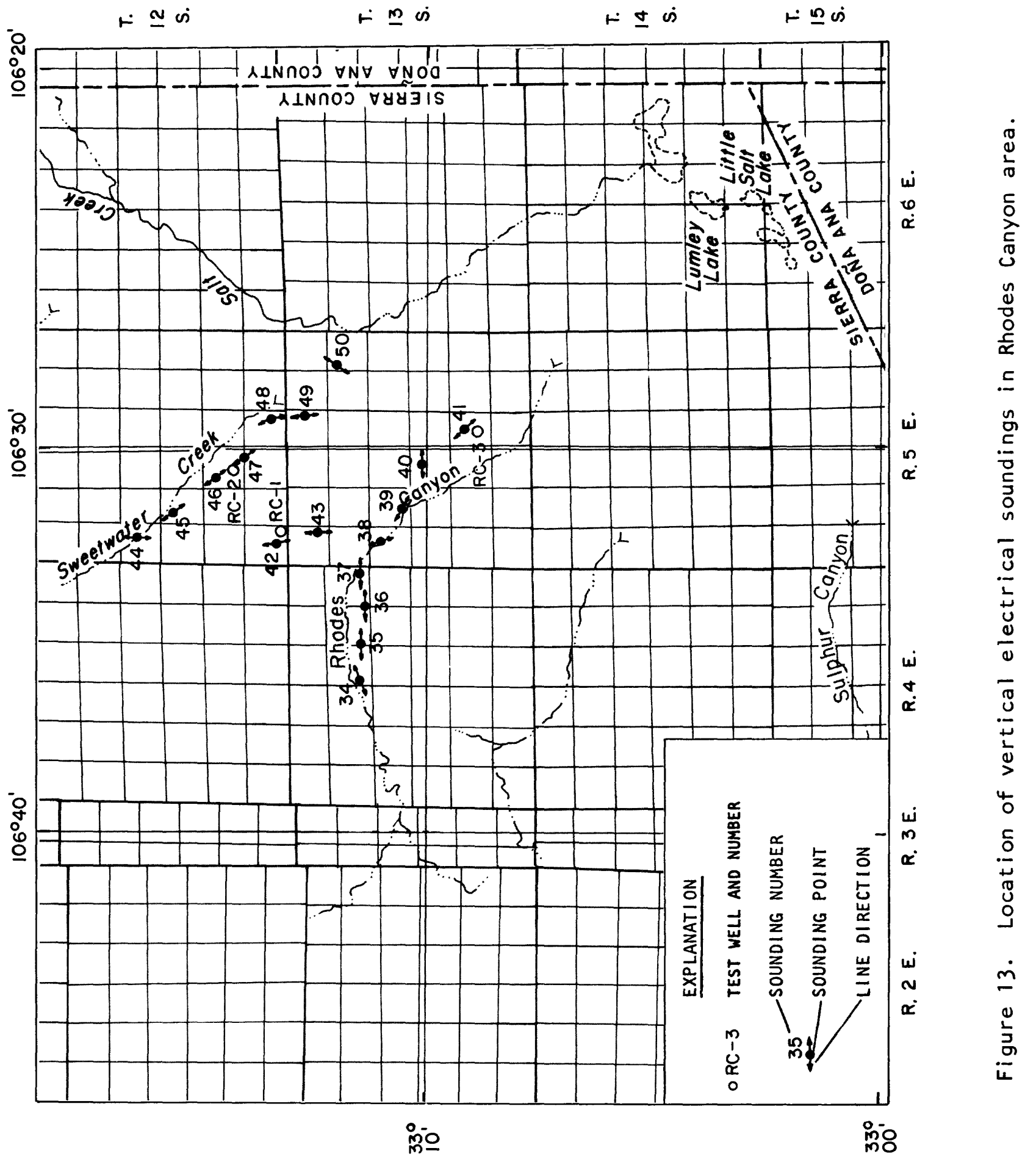




\section{SELECTED REFERENCES}

Cooper, J. B., 1970, Summary records of supply wells and test wells in the Post Headquarters area, White Sands Missile Range, New Mexico: U.S. Geological Survey open-file report, 202 p.

1973, Summary records of test and supply wells in Range Areas, White Sands Missile Range, New Mexico: U.S. Geological Survey open-file report, $132 \mathrm{p}$.

Cruz, R. R., 1982, Annual water-resources review, White Sands Missile Range, New Mexico, 1981: U.S. Geological Survey Open-File Report 82-757, 20 p.

Ke11y, T. E., 1973, Summary of ground-water data at Post Headquarters and adjacent areas, White Sands Missile Range: U.S. Geological Survey openfile report, $66 \mathrm{p}$.

Wier, J. E., Jr., 1965, Geology and availability of ground water in the northern part of White Sands Missile Range and vicinity, New Mexico: U.S. Geological Survey Water-Supply Paper 1801, 78 p. 


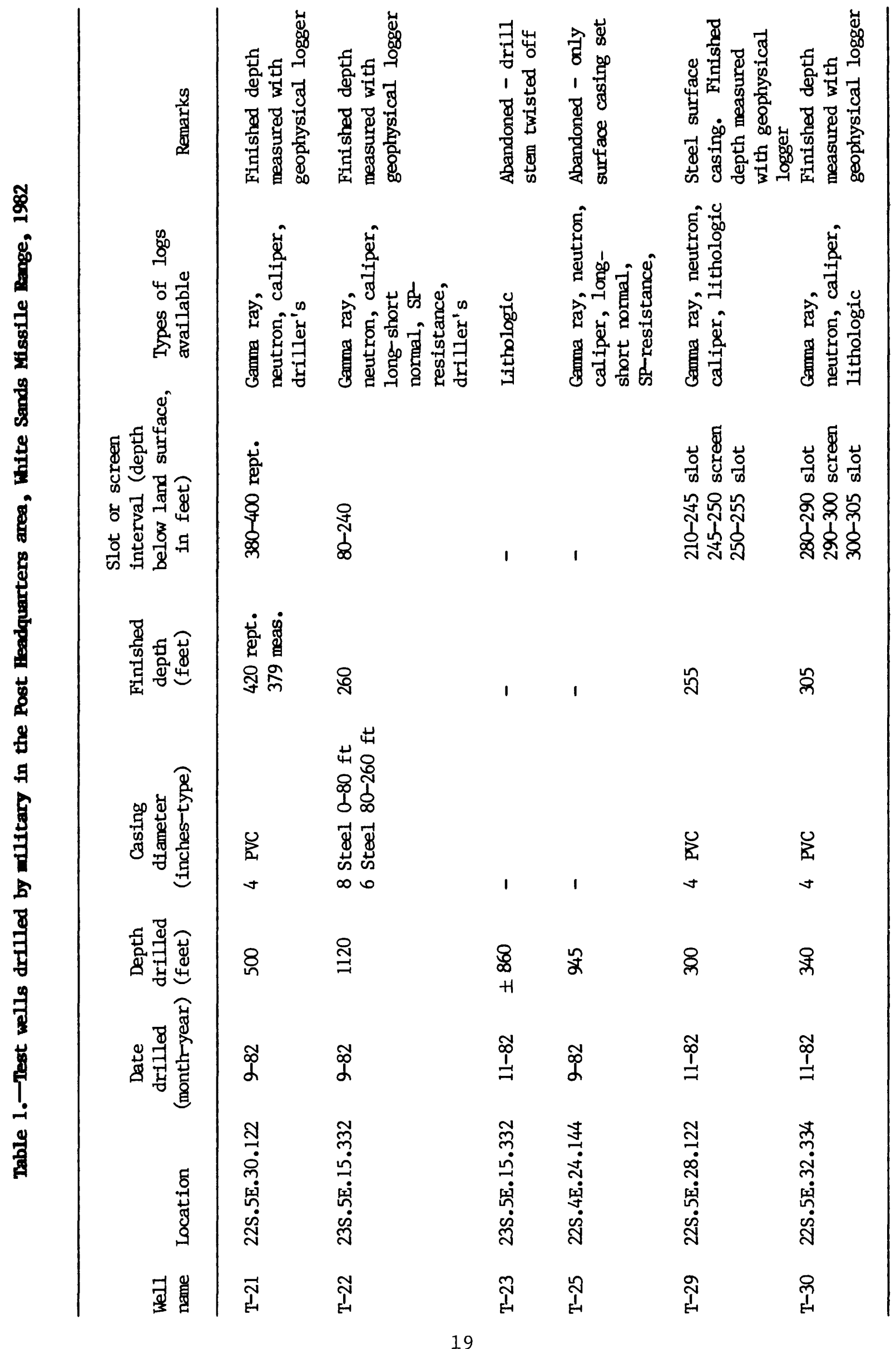


Table 2.-Depth to water in supply wells, Post Headquarters and

Range areas, 1982

\begin{tabular}{|c|c|c|c|}
\hline $\begin{array}{l}\text { We11 } \\
\text { number }\end{array}$ & Location & $\begin{array}{l}\text { March } 1982 \\
\text { (feet below } \\
\text { land surface) }\end{array}$ & $\begin{array}{l}\text { August } 1982 \\
\text { (feet below } \\
\text { land surface) }\end{array}$ \\
\hline $10 \mathrm{~A}$ & $22 \mathrm{~S} .4 \mathrm{E} \cdot 24.212 \mathrm{a}$ & 426.59 & 436.49 \\
\hline 11 & $22 \mathrm{~S} .4 \mathrm{E} \cdot 24.112$ & $265.20 *$ & $365.00 *$ \\
\hline 13 & $22 \mathrm{~S} .4 \mathrm{E} \cdot 13.311$ & 298.96 & 299.61 \\
\hline 15 & $22 \mathrm{~S} .4 \mathrm{E} .13: 424$ & - & 433.97 \\
\hline 16 & $22 \mathrm{~S} .4 \mathrm{E} \cdot 13.432$ & $424.85 *$ & $467.00 *$ \\
\hline 17 & $22 \mathrm{~S} .4 \mathrm{E} .13 .241$ & 437.64 & 469.75 \\
\hline 18 & $22 \mathrm{~S} .4 \mathrm{E} .12 .434$ & 428.94 & 450.93 \\
\hline 19 & $22 \mathrm{~S} .4 \mathrm{E} .12 .414$ & 452.79 & 460.96 \\
\hline 20 & $22 \mathrm{~S} .4 \mathrm{E} \cdot 12.214$ & 512.99 & 519.47 \\
\hline 21 & $22 \mathrm{~S} .5 \mathrm{E} \cdot 19.323$ & 354.62 & 360.19 \\
\hline 22 & $22 \mathrm{~S} .5 \mathrm{E} .19 .414$ & 374.24 & 389.07 \\
\hline HTA-1 & $21 \mathrm{~S} .4 \mathrm{E} .23 .233$ & 62.32 & 64.07 \\
\hline SMR-1 & $21 \mathrm{~S} .5 \mathrm{E} .16 .132$ & 294.78 & 295.79 \\
\hline MAR-1 & $19 \mathrm{~S} .5 \mathrm{E} .17 .331$ & - & 223.29 \\
\hline MAR-2 & 19S.5E.17.334 & 221.20 & 220.92 \\
\hline SRC-1 & $6 \mathrm{~S} \cdot 3 \mathrm{E} \cdot 05 \cdot 232$ & 207.70 & 208.80 \\
\hline SRC-2 & $6 \mathrm{~S} \cdot 3 \mathrm{E} \cdot 05 \cdot 234$ & 212.65 & 212.65 \\
\hline
\end{tabular}

* Air line reading 
Table 3.-Depth to water in test and observation wells, Post Headquarters and Range areas, 1982

\begin{tabular}{|c|c|c|c|}
\hline $\begin{array}{l}\text { We11 } \\
\text { number }\end{array}$ & Location & $\begin{array}{l}\text { March } 1982 \\
\text { (feet below } \\
\text { land surface) }\end{array}$ & $\begin{array}{l}\text { August } 1982 \\
\text { (feet below } \\
\text { land surface) }\end{array}$ \\
\hline $\mathrm{T}-4$ & $22 \mathrm{~S} .5 \mathrm{E} .16 .111$ & 226.42 & 226.57 \\
\hline $\mathrm{T}-5$ & $22 S \cdot 5 E \cdot 20.111$ & 276.42 & 276.52 \\
\hline $\mathrm{T}-6$ & $22 \mathrm{~S} .4 \mathrm{E} .14 .133$ & 199.59 & 198.04 \\
\hline $\mathrm{T}-7$ & $22 \mathrm{~S} \cdot 5 \mathrm{E} \cdot 07 \cdot 342$ & 358.43 & 375.04 \\
\hline $\mathrm{T}-8$ & $22 \mathrm{~S} .4 \mathrm{E} .11 .224$ & 580.50 & 582.91 \\
\hline $\mathrm{T}-9$ & $22 \mathrm{~S} .4 \mathrm{E} .01 .431$ & 389.32 & 384.98 \\
\hline $\mathrm{T}-10$ & $22 \mathrm{~S} .5 \mathrm{E} .03 .313$ & 272.03 & 272.32 \\
\hline $\mathrm{T}-11$ & $22 \mathrm{~S} .5 \mathrm{E} .29 \cdot 412$ & 272.68 & 272.78 \\
\hline $\mathrm{T}-13$ & $22 \mathrm{~S} \cdot 5 \mathrm{E} \cdot 32 \cdot 222$ & 212.79 & 212.79 \\
\hline $\mathrm{T}-14$ & $22 \mathrm{~S} \cdot 5 \mathrm{E} \cdot 15.221$ & 132.13 & 132.13 \\
\hline $\mathrm{T}-15$ & $22 \mathrm{~S} \cdot 5 \mathrm{E} \cdot 33.244$ & 179.16 & 179.63 \\
\hline $\mathrm{T}-16$ & $23 \mathrm{~S} \cdot 5 \mathrm{E} \cdot 10 \cdot 413$ & 185.07 & 184.94 \\
\hline $\mathrm{T}-17$ & $23 \mathrm{~S} \cdot 5 \mathrm{E} \cdot 27 \cdot 142$ & 246.65 & 242.43 \\
\hline $\mathrm{T}-18$ & $23 \mathrm{~S} .5 \mathrm{E} .05 .321$ & 239.99 & 238.63 \\
\hline os -9 & $22 \mathrm{~S} .5 \mathrm{E} \cdot 31.424$ & 244.00 & 243.89 \\
\hline os-12 & $22 \mathrm{~S} \cdot 4 \mathrm{E} \cdot 23.214$ & 231.92 & 232.74 \\
\hline Gregg & $22 \mathrm{~S} .6 \mathrm{E} .08 .414$ & 214.24 & 214.52 \\
\hline HTA（wm） & $21 \mathrm{~S} \cdot 4 \mathrm{E} \cdot 22.222$ & 42.88 & 41.88 \\
\hline$S M R-2$ & $21 \mathrm{~S} \cdot 5 \mathrm{E} \cdot 17.424$ & 320.19 & 320.25 \\
\hline SMR-3 & $20 \mathrm{~S} \cdot 5 \mathrm{E} \cdot 34.133$ & 294.92 & 295.47 \\
\hline$S M R-4$ & $21 S \cdot 5 E \cdot 20 \cdot 344$ & 287.90 & 288.18 \\
\hline MAR-1 (test) & $19 \mathrm{~S} .5 \mathrm{E} .17 .333$ & 221.39 & 221.29 \\
\hline$M A R-4$ & $19 S .5 E .19 .231$ & 302.96 & 303.10 \\
\hline NW30-1 & $17 \mathrm{~S} .4 \mathrm{E} .02 .211$ & 212.54 & 212.65 \\
\hline Murray & $8 S \cdot 5 E \cdot 32 \cdot 334$ & 176.94 & 177.38 \\
\hline Lucero Ranch & $19 S \cdot 5 E \cdot 22 \cdot 334$ & 169.60 & 171.05 \\
\hline $\mathrm{CW}$ & $21 \mathrm{~S} .5 \mathrm{E} .28 .311$ & - & 153.52 \\
\hline
\end{tabular}


Table 4.-Depth to water in boreholes, Post Headquarters and adjacent areas, 1982

\begin{tabular}{|c|c|c|c|}
\hline $\begin{array}{l}\text { We11 } \\
\text { number }\end{array}$ & Location & $\begin{array}{l}\text { March } 1982 \\
\text { (feet below } \\
\text { land surface) }\end{array}$ & $\begin{array}{l}\text { August } 1982 \\
\text { (feet below } \\
\text { land surface) }\end{array}$ \\
\hline$B-1$ & $22 \mathrm{~S} .05 \mathrm{E} .28 .122$ & 192.29 & - \\
\hline $\mathrm{B}-2$ & $22 \mathrm{~S} .05 \mathrm{E} .28 .124$ & 195.81 & 195.44 \\
\hline$B-3$ & $22 \mathrm{~S} .05 \mathrm{E} .28 .142$ & 202.99 & 203.03 \\
\hline$B-4$ & $22 \mathrm{~S} .05 \mathrm{E} .28 .233$ & 197.29 & 197.54 \\
\hline$B-5$ & $22 \mathrm{~S} .05 \mathrm{E} .33 .223$ & 187.79 & 187.87 \\
\hline$B-6$ & $23 \mathrm{~S} .05 \mathrm{E} .01 .113$ & 133.78 & 133.10 \\
\hline$B-9$ & $22 \mathrm{~S} .05 \mathrm{E} .21 .211$ & 225.20 & 225.34 \\
\hline $\mathrm{B}-10$ & $22 \mathrm{~S} .05 \mathrm{E} .19 .414$ & 305.55 & 306.34 \\
\hline$B-13$ & $22 \mathrm{~S} .05 \mathrm{E} .08 .141$ & 242.68 & 243.20 \\
\hline$B-14$ & $22 \mathrm{~S} .05 \mathrm{E} .03 .221$ & 112.20 & 112.30 \\
\hline$B-15$ & $22 \mathrm{~S} .05 \mathrm{E} .05 .242$ & 172.98 & 173.62 \\
\hline $\mathrm{B}-16$ & $21 \mathrm{~S} .05 \mathrm{E} .34 .213$ & 109.18 & 109.30 \\
\hline $\mathrm{B}-17$ & $21 \mathrm{~S} .05 \mathrm{E} .33 .242$ & 111.39 & 111.46 \\
\hline$B-18$ & $21 \mathrm{~S} .05 \mathrm{E} .23 .134$ & 104.21 & 104.33 \\
\hline$B-20$ & $22 \mathrm{~S} .04 \mathrm{E} .14 .134$ & 347.80 & 349.20 \\
\hline $\mathrm{B}-23$ & $22 \mathrm{~S} .05 \mathrm{E} .16 .111$ & 224.75 & 224.82 \\
\hline$B-26$ & $21 \mathrm{~S} .06 \mathrm{E} .32 .114$ & 141.07 & 141.21 \\
\hline$B-27$ & $21 \mathrm{~s} .06 \mathrm{E} .17 .314$ & 119.78 & 119.81 \\
\hline$B-28$ & $21 \mathrm{~s} .05 \mathrm{E} .02 .341$ & 140.67 & 140.26 \\
\hline $\mathrm{B}-30$ & $20 \mathrm{~S} .05 \mathrm{E} .23 .213$ & 89.64 & 89.67 \\
\hline
\end{tabular}


Table 4.-Depth to water in boreholes, Post Headquarters and adjacent areas, 1982 - Concluded

\begin{tabular}{|c|c|c|c|}
\hline $\begin{array}{l}\text { We11 } \\
\text { number }\end{array}$ & Location & $\begin{array}{l}\text { March } 1982 \\
\text { (feet below } \\
\text { land surface) }\end{array}$ & $\begin{array}{l}\text { August } 1982 \\
\text { (feet below } \\
\text { land surface) }\end{array}$ \\
\hline$B-31$ & $20 \mathrm{~S} .06 \mathrm{E} \cdot 29.123$ & 123.30 & 123.41 \\
\hline$B-34$ & $21 \mathrm{~S} .05 \mathrm{E} .01 .221$ & 126.24 & 126.29 \\
\hline$B-36$ & $22 \mathrm{~S} .04 \mathrm{E} .01 .323$ & 211.09 & 211.40 \\
\hline$B-37$ & $22 \mathrm{~S} .04 \mathrm{E} .11 .344$ & 396.32 & 394.70 \\
\hline B-38 & $20 \mathrm{~S} .06 \mathrm{E} \cdot 11.234$ & 129.76 & 129.85 \\
\hline$B-39$ & $21 \mathrm{~S} .06 \mathrm{E} .02 .142$ & 156.31 & 156.37 \\
\hline$B-40$ & $21 \mathrm{~S} .06 \mathrm{E} \cdot 26.142$ & 188.60 & 188.56 \\
\hline$B-42$ & $22 \mathrm{~S} .04 \mathrm{E} .11 .444$ & 379.64 & 378.78 \\
\hline$B-46$ & $21 \mathrm{~S} .05 \mathrm{E} .27 .113$ & 135.67 & 135.70 \\
\hline$B-47$ & $22 \mathrm{~S} .05 \mathrm{E} .08 .334$ & 273.29 & 273.92 \\
\hline$B-48$ & $22 \mathrm{~S} \cdot 06 \mathrm{E} \cdot 31 \cdot 322$ & 204.47 & 204.57 \\
\hline$B-49$ & $22 \mathrm{~S} .05 \mathrm{E} .09 .113$ & 199.39 & 199.70 \\
\hline$B-50$ & $22 \mathrm{~S} .05 \mathrm{E} .07 .242$ & 304.87 & 305.25 \\
\hline$B-51$ & $22 \mathrm{~S} .05 \mathrm{E} .26 .312$ & 146.68 & 146.64 \\
\hline$B-52$ & $22 \mathrm{~S} .05 \mathrm{E} .09 .113$ & 210.29 & 210.62 \\
\hline B-54 & $22 \mathrm{~S} .05 \mathrm{E} .16 .111$ & 229.98 & 229.79 \\
\hline$B-55$ & $22 \mathrm{~S} .05 \mathrm{E} .09 .113$ & 214.14 & 214.70 \\
\hline$B-56$ & $22 \mathrm{~S} .05 \mathrm{E} .30 .424$ & 279.40 & 276.86 \\
\hline
\end{tabular}


Table 5.-Specific-conductance values of water samples collected from supply wells, test wells, and observation wells, 1982

Part I. Supply wells

\begin{tabular}{|c|c|c|}
\hline \multirow{2}{*}{$\begin{array}{l}\text { We11 } \\
\text { number }\end{array}$} & \multicolumn{2}{|c|}{$\begin{array}{l}\text { Specific-conductance value (laboratory) } \\
\text { (micromhos per centimeter at } 25^{\circ} \mathrm{C} \text { ) }\end{array}$} \\
\hline & Winter & Summer \\
\hline $10 \mathrm{~A}$ & 477 & 331 \\
\hline 11 & 683 & 403 \\
\hline 13 & 611 & 600 \\
\hline 16 & - & 385 \\
\hline 17 & 545 & 372 \\
\hline 18 & 564 & 389 \\
\hline 19 & 392 & 392 \\
\hline 20 & 572 & 584 \\
\hline 21 & 288 & 290 \\
\hline 22 & - & - \\
\hline HTA-1 & 1020 & 718 \\
\hline SMR-1 & 1130 & 781 \\
\hline MAR-1 & - & 906 \\
\hline MAR-2 & 1120 & - \\
\hline SRC-1 & 3480 & 3430 \\
\hline SRC-2 & 3450 & - \\
\hline $\begin{array}{l}\text { SRC ( product } \\
\text { water) }\end{array}$ & 381 & 456 \\
\hline
\end{tabular}


Table 5.-Specific-conductance values of water samples collected from supp1y wells, test wells, and observation we11s, 1982 - Concluded

Part II. Test and observation wells

\begin{tabular}{|c|c|c|}
\hline $\begin{array}{l}\text { We11 } \\
\text { number }\end{array}$ & $\begin{array}{l}\text { Specific conductance value ( } 1 \mathrm{ab} \text { ) } \\
\text { (micromhos per centimeter at } 25^{\circ} \mathrm{C} \text { ) } \\
\text { (samples collected during summer) }\end{array}$ & $\begin{array}{l}\text { Sampling point } \\
\text { (feet below } \\
\text { land surface) }\end{array}$ \\
\hline$T-4$ & 317 & 325 \\
\hline$T-5$ & 371 & 330 \\
\hline $\mathrm{T}-7$ & 344 & 440 \\
\hline $\mathrm{T}-7$ & 441 & 960 \\
\hline $\mathrm{T}-8$ & 680 & 610 \\
\hline $\mathrm{T}-8$ & 626 & 915 \\
\hline $\mathrm{T}-9$ & 883 & 550 \\
\hline $\mathrm{T}-10$ & 331 & 530 \\
\hline $\mathrm{T}-13$ & 490 & 410 \\
\hline $\mathrm{T}-14$ & 1498 & 200 \\
\hline $\mathrm{T}-14$ & 1479 & 300 \\
\hline $\mathrm{T}-15$ & 323 & 448 \\
\hline $\mathrm{T}-16$ & 289 & 480 \\
\hline $\mathrm{T}-17$ & 167 & 440 \\
\hline $\mathrm{T}-18$ & 703 & 635 \\
\hline $0 s-12$ & 440 & 340 \\
\hline Lucero Ranch & 840 & 210 \\
\hline
\end{tabular}




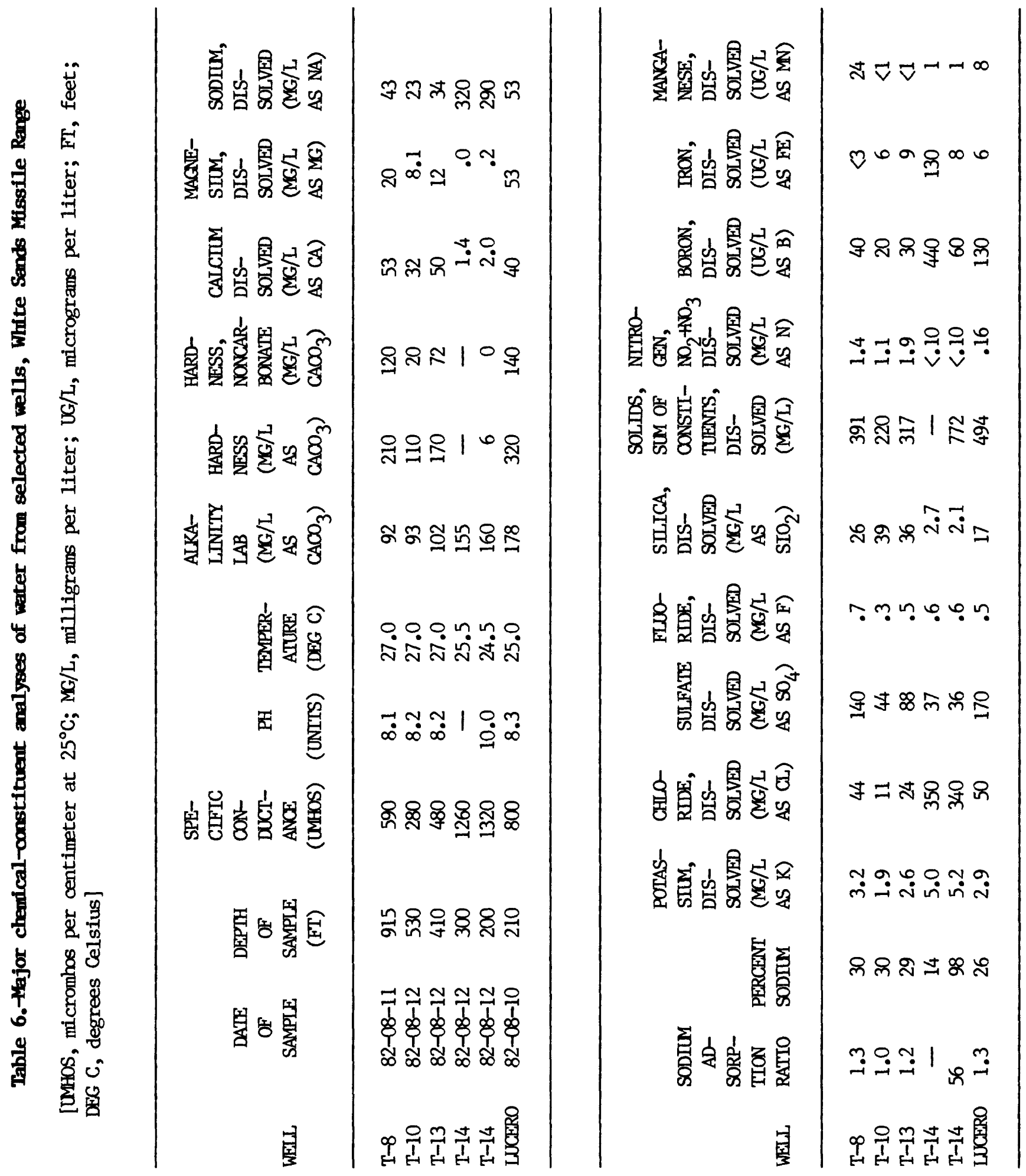




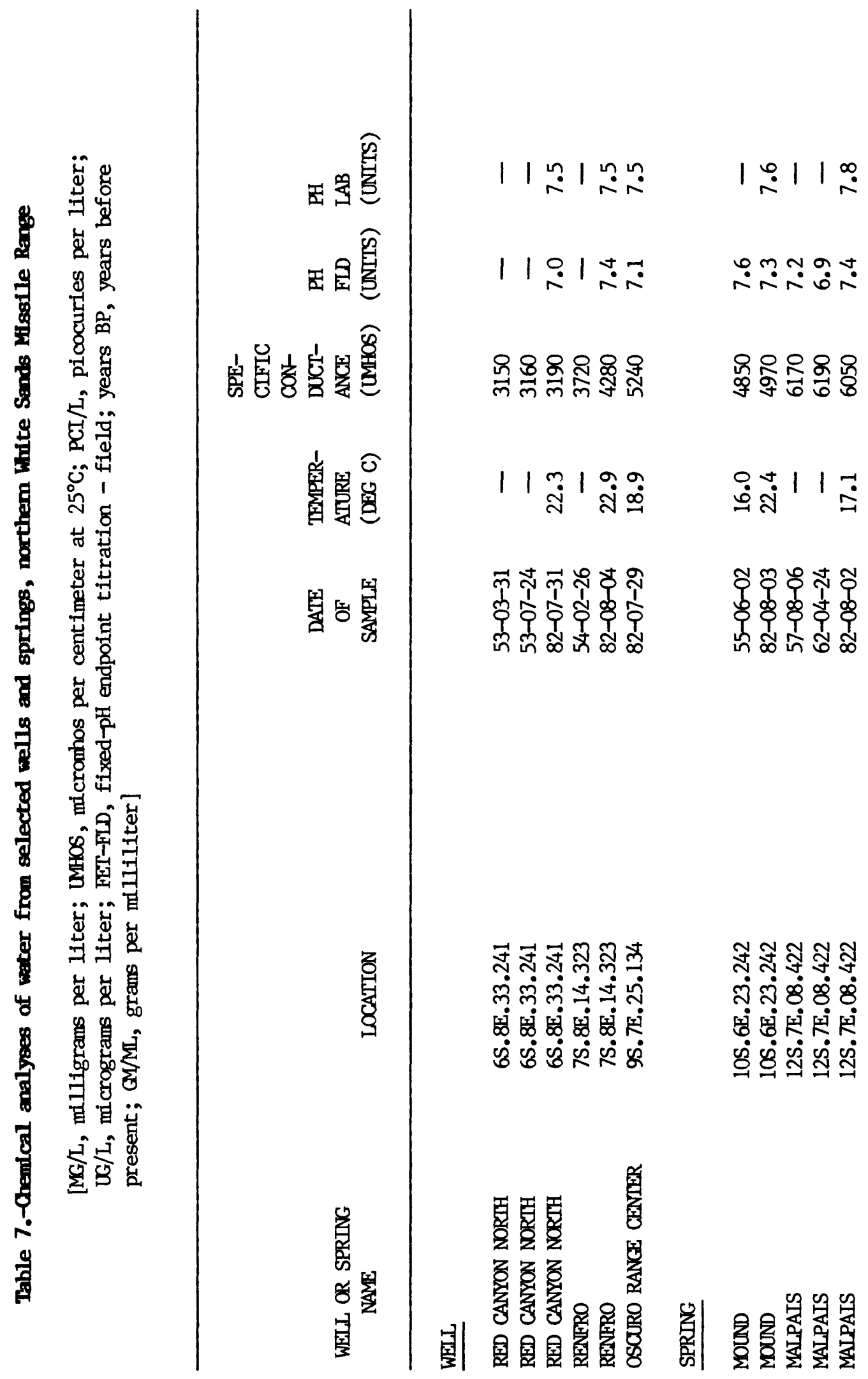




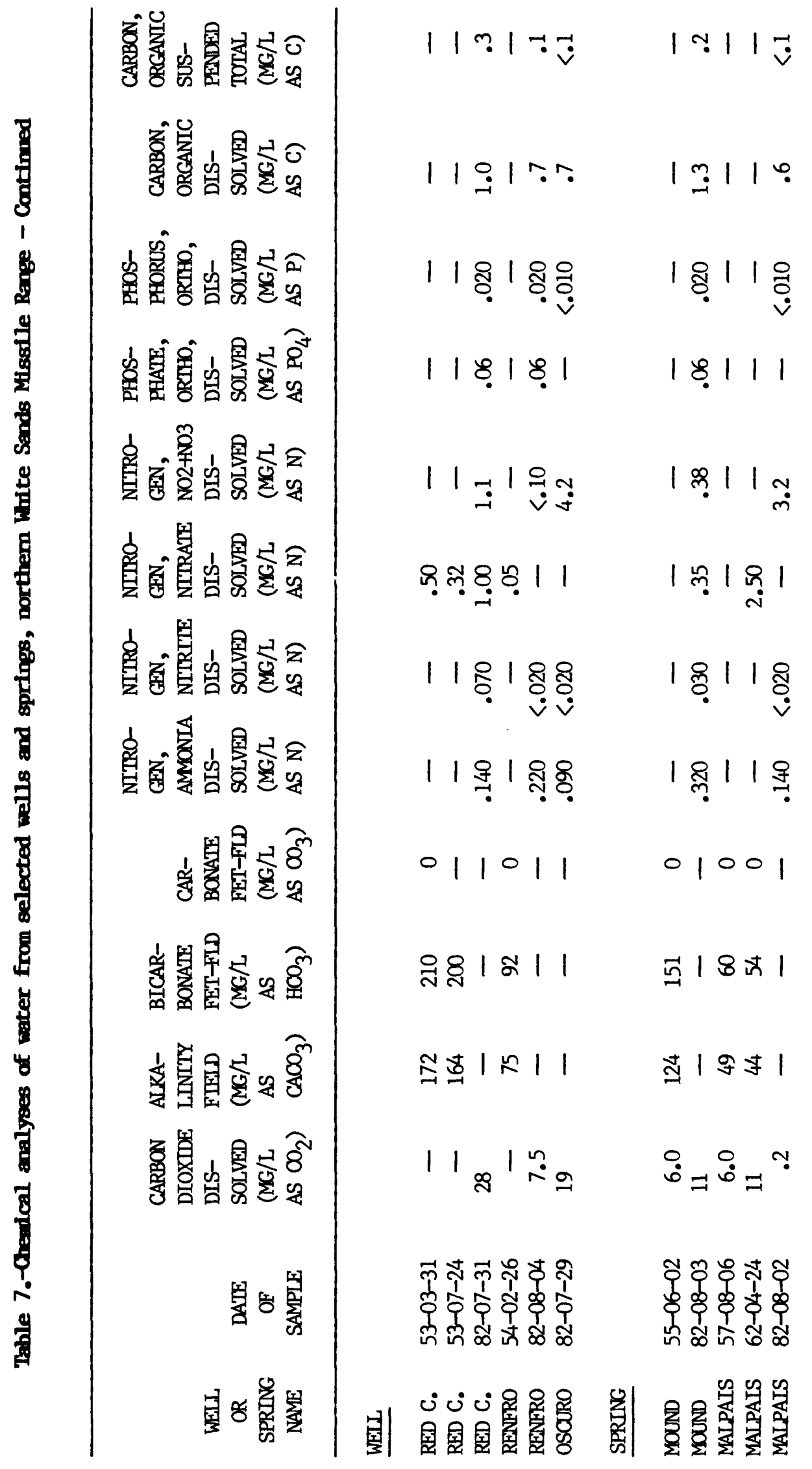




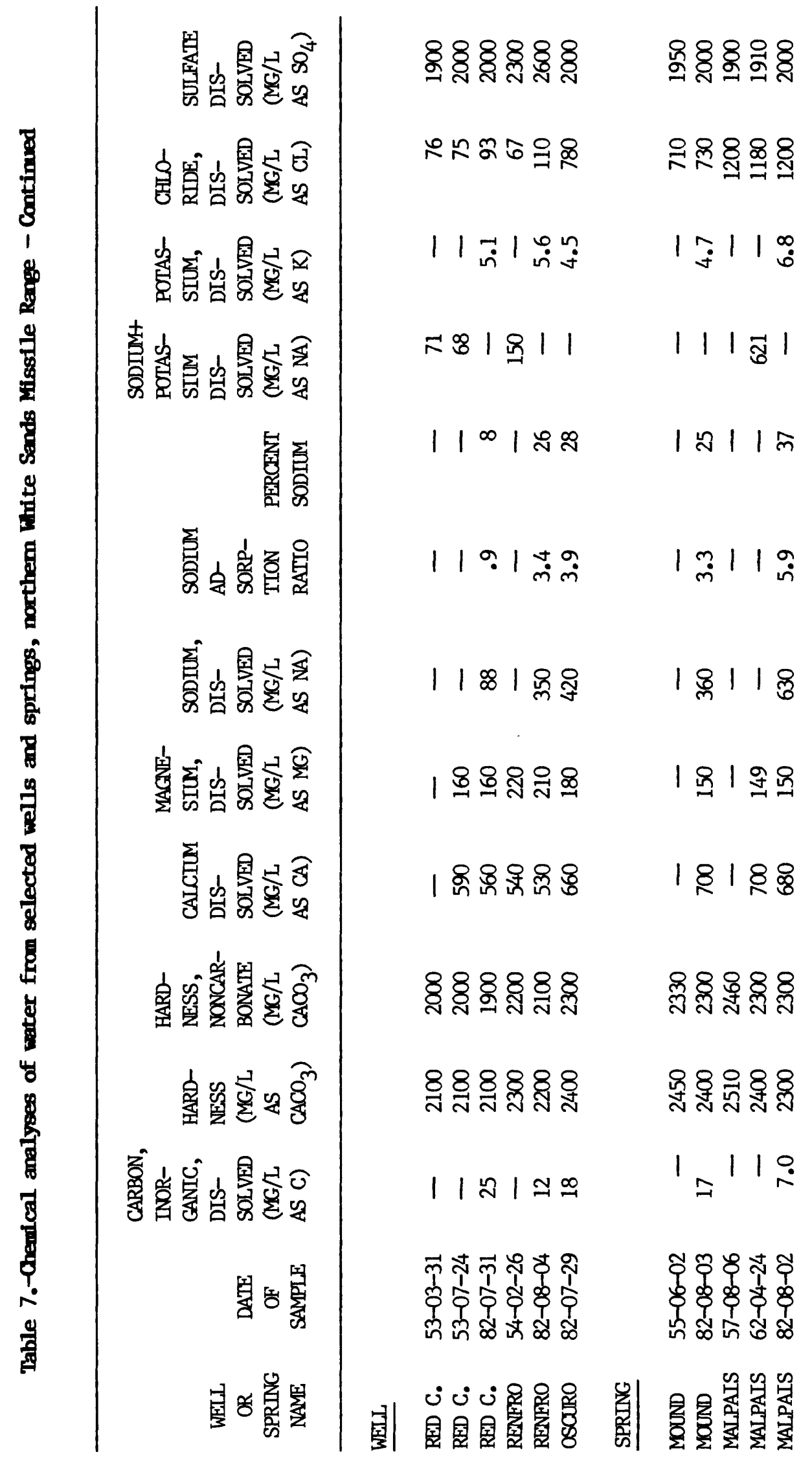




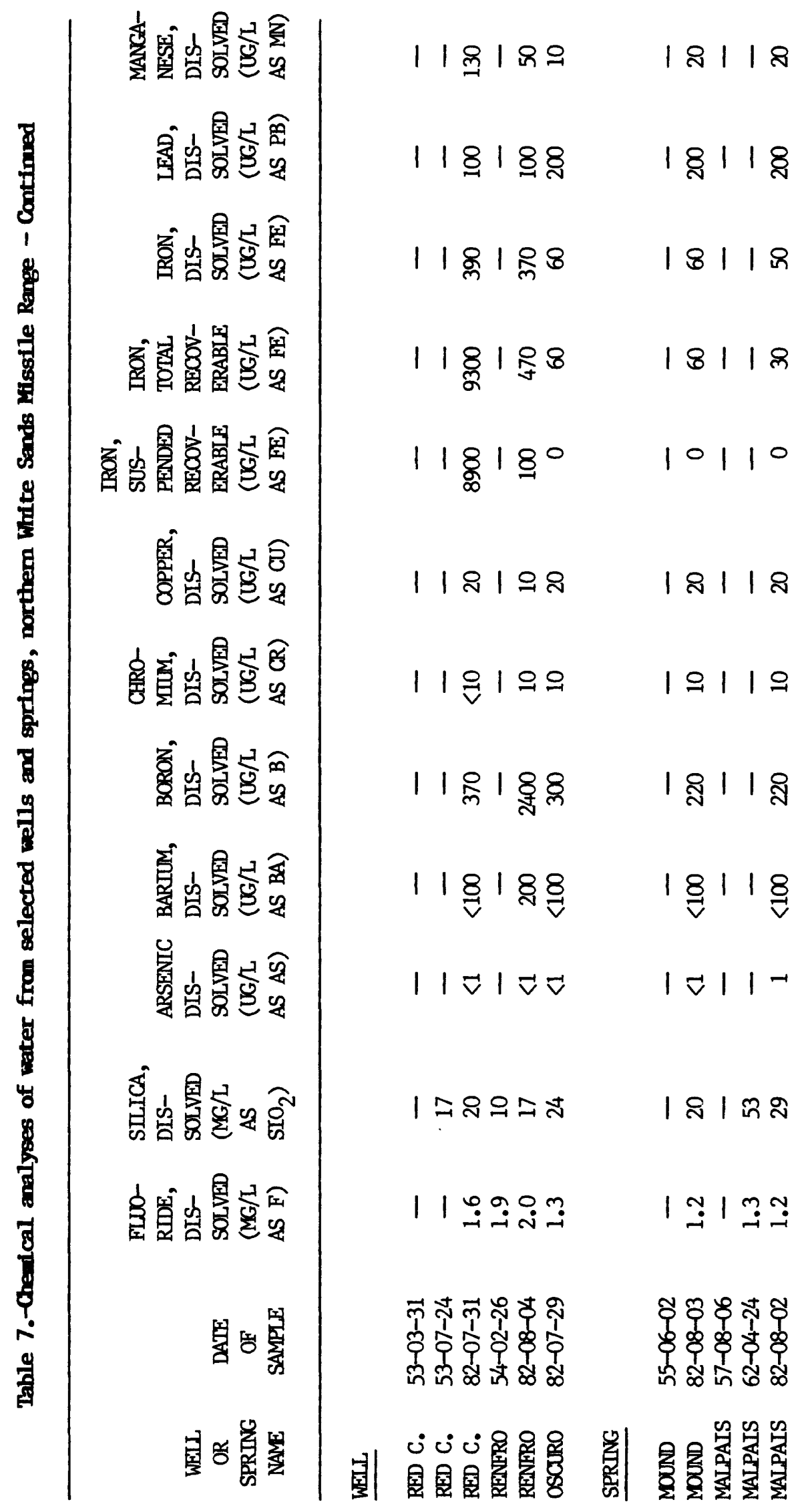




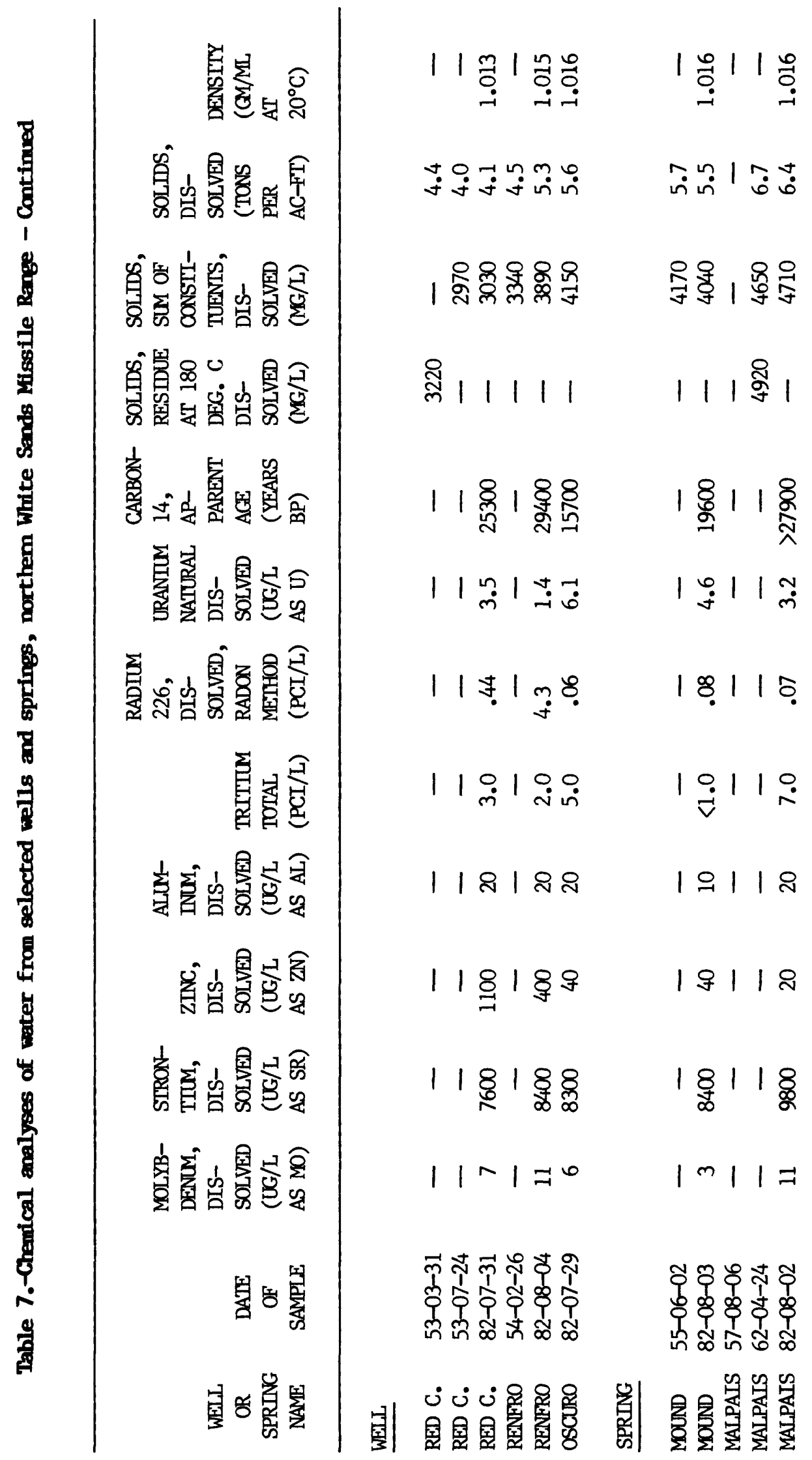




\begin{tabular}{|c|c|c|}
\hline 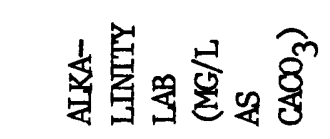 & $119.18 \mathrm{I}$ & 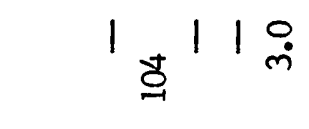 \\
\hline 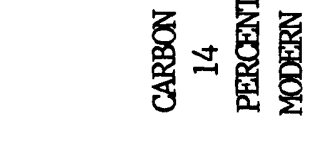 & 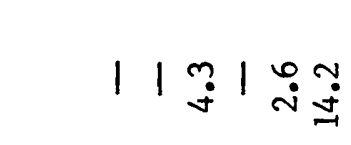 & $\left|\begin{array}{ll}\mid \infty \\
\infty\end{array}\right| \begin{array}{l}\mid \\
\infty\end{array} \mid \overrightarrow{0}$ \\
\hline 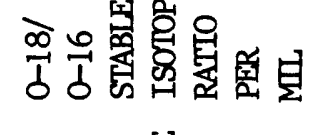 & 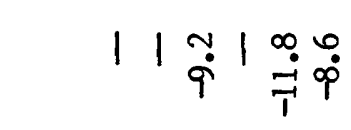 & 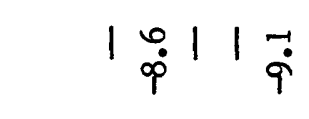 \\
\hline 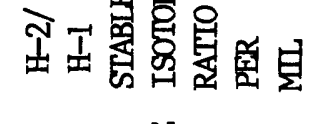 & 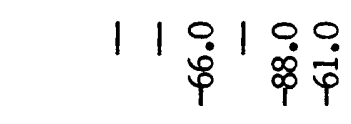 & $1 \underset{\dot{\varphi}}{n} 110$ \\
\hline 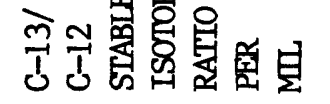 & 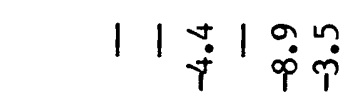 & 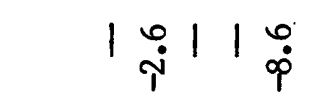 \\
\hline & 11 1 19 & $|\stackrel{1}{1}| 1$. \\
\hline 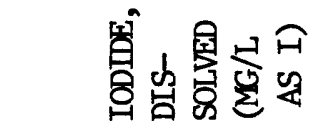 & 118 & $18.11 \%$ \\
\hline 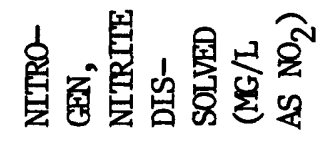 & | 19.111 & 19111 \\
\hline 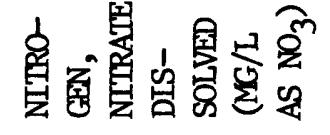 & 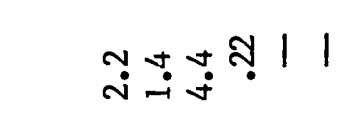 & $\ln _{-1} 1=1$ \\
\hline 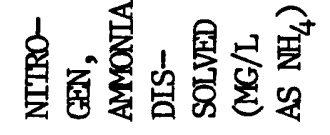 & $|1 \stackrel{9}{1}| \stackrel{9}{9} \div$ & $|\exists .1| \stackrel{\infty}{.}$ \\
\hline 圈四罢 & 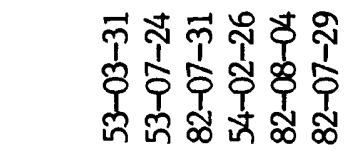 & 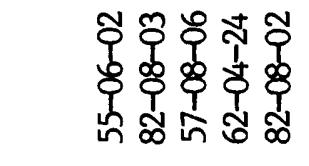 \\
\hline 自安总累 & 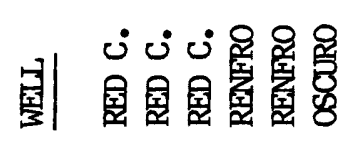 & 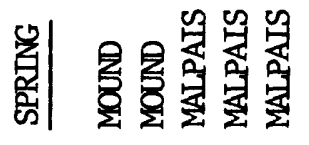 \\
\hline
\end{tabular}

